\title{
Walsh-synthesized noise filters for quantum logic
}

\author{
Harrison Ball ${ }^{1,2}$ and Michael J Biercuk ${ }^{1,2^{*}}$
}

\author{
"Correspondence: \\ michael.biercuk@sydney.edu.au \\ ${ }^{1}$ ARC Centre for Engineered \\ Quantum Systems, School of \\ Physics, The University of Sydney, \\ Sydney, NSW 2006, Australia \\ ${ }^{2}$ National Measurement Institute, \\ West Lindfield, Sydney, NSW 2070, \\ Australia
}

\begin{abstract}
We study a novel class of open-loop control protocols constructed to perform arbitrary nontrivial single-qubit logic operations robust against time-dependent non-Markovian noise. Amplitude and phase modulation protocols are crafted leveraging insights from functional synthesis and the basis set of Walsh functions. We employ the experimentally validated generalized filter-transfer function formalism in order to find optimized control protocols for target operations in SU(2) by defining a cost function for the filter-transfer function to be minimized through the applied modulation. Our work details the various techniques by which we define and then optimize the filter-synthesis process in the Walsh basis, including the definition of specific analytic design rules which serve to efficiently constrain the available synthesis space. This approach yields modulated-gate constructions consisting of chains of discrete pulse-segments of arbitrary form, whose modulation envelopes possess intrinsic compatibility with digital logic and clocking. We derive novel families of Walsh-modulated noise filters designed to suppress dephasing and coherent amplitude-damping noise, and describe how well-known sequences derived in NMR also fall within the Walsh-synthesis framework. Finally, our work considers the effects of realistic experimental constraints such as limited modulation bandwidth on achievable filter performance.
\end{abstract}

Keywords: decoherence suppression; error correction; open-loop control; dynamic error suppression; quantum control; quantum logic; qubit; Walsh function; functional analysis

\section{Introduction}

In realistic laboratory settings, decoherence in quantum systems is dominated by timedependent non-Markovian noise processes with long correlations, frequently characterized by low-frequency dominated noise power spectra [1-5]. These may arise either from environmental fluctuations or - in the important case of driven quantum systems - from noise in the control device itself [6]. In either case, the result is a reduction in the fidelity of a target control operation, including both memory and nontrivial operations. These phenomena present a major challenge as quantum devices move from proof of principle demonstrations to realistic applications, where performance demands on the quantum devices are frequently extreme. Accordingly, finding ways to control quantum systems efficiently and effectively in the presence of noise is a central task in quantum control theory [7-10].

(C) 2015 Ball and Biercuk; licensee Springer. This article is distributed under the terms of the Creative Commons Attribution 4.0 International License (http://creativecommons.org/licenses/by/4.0/), which permits unrestricted use, distribution, and reproduction in any medium, provided you give appropriate credit to the original author(s) and the source, provide a link to the Creative Commons license, and indicate if changes were made. 
A range of techniques relying on both open- and closed-loop control have been devised to address this challenge [11-13] at various levels in a layered architecture for quantum computing [14]. In particular, open-loop dynamical error suppression strategies (without the need for measurement or feedback) such as dynamic decoupling (DD) [15-18], dynamically corrected gates (DCGs) [19-25], and composite pulsing [26-28], have emerged as resource-efficient approaches for physical-layer decoherence control. They are joined by a new class of continuously modulated ('always-on') dynamical decoupling and dynamically protected gate schemes [29-36] inspired by well established techniques in NMR.

These schemes all address the question of decoherence mitigation, but looking across their breadth, have both benefitted and suffered from reliance on a wide range of theoretical techniques. Unfortunately the analytic tools for crafting control protocols employed in any particular setting do not necessarily translate equivalently between approaches, nor do the methods generally employed for evaluating efficacy easily translate to experimentally measured characteristics of the environment. This is a major challenge for experimentalists or systems designers attempting to determine which of the many openloop control schemes to employ in a particular experiment. As an example, the powerful group theoretic insights and consideration of time-varying environments that permit the construction of error-robust, bounded-strength $\mathrm{SU}(2)$ operations for quantum information in Viola's DCG framework are quite different from the geometric considerations and quasi-static noise assumptions widely employed in NMR composite pulsing. This issue has been highlighted recently as new work has revealed striking differences between the time-domain noise sensitivity of control protocols as compared to longstanding notions of error cancellation in the Magnus expansion [37, 38].

A unified and experimentally relevant framework for devising and evaluating errorsuppressing gates in realistic noise environments is therefore needed to secure the role of dynamical error suppression in systematic designs of quantum technologies including fault-tolerant quantum computers. Kurizki provided a promising path towards this end with his seminal work framing the problem of finding decoherence-suppressing control protocols by considering appropriate frequency-domain modification of the systemenvironment coupling $[39,40]$. Residual errors could be calculated through overlap integrals of the power spectrum describing the environmental noise, and functions capturing the frequency-domain response of any applied control. This framework - effectively a quantum generalization of transfer functions widely used in control engineering [41] - provides a simple heuristic approach to understanding the performance of an arbitrary control protocol in an arbitrary noise environment. Stated simply, effective error-suppressing control protocols 'filter' the noise over a user-defined band, therefore mitigating decoherence in the quantum system [42].

Early demonstrations of this framework applied to the simple case of implementing the protected identity operator to qubits by dynamical decoupling [15, 43-47], where the filter functions could be calculated for pure dephasing in a straightforward manner using concepts of linear control [42]. Expanding significantly beyond this work, the challenge of crafting generalized analytic forms for the transfer functions describing arbitrary singlequbit control compatible with universal non-commuting noise (a problem in nonlinear control) has recently been addressed theoretically [28, 38, 48, 49], and validated in experiment [37]. Further theoretical extensions of filter-transfer functions to two-qubit gates highlight the breadth of applicability of this approach to quantum control [50-52]. 
Beyond its simple intuitive nature, the power of the filter transfer function approach comes from the fact that it can in principle be applied to studying dynamic-errorsuppression control protocols derived through any manner of analytic approach. It permits the application of well tested engineering concepts for control systems design; the complex physics associated with quantum dynamics in time-dependent environments with non-commuting noise and control Hamiltonians is relegated to the calculation of the generalized filter transfer functions themselves, and once derived these may be deployed in block-diagram systems analyses [41].

With these significant advances and the promise of applying the suite of insights from control theory to the quantum regime, the noise-filtering approach to quantum control has leapt to the fore, providing a unifying framework applicable over a wide parameter range of interest to real experimental settings. Nonetheless, outstanding challenges remain in how to leverage the generalized filter-transfer-function framework $[48,49]$ to systematically craft effective error-suppressing gate constructions while also heeding realistic system constraints imposed by hardware systems. For instance, the presence of finite timing precision and limited classical communication bandwidth between the physical (quantum) layer and a classical controller [14] impose new constraints not generally captured when solely considering quantum dynamical evolution of an individual state.

We address this challenge, introducing a quantum control toolkit permitting the realization of physical-layer error-suppressing control protocols that are simultaneously effective in suppressing error and compatible with a variety of major hardware restrictions. We leverage the generalized filter-transfer function formalism as a unifying theoretical construct, and employ techniques from functional analysis in order to realize appropriate modulation protocols applied to a near-resonant carrier frequency for enacting highfidelity quantum control operations on single qubits [30,53]. Our work identifies the Walsh functions - square-wave analogues of the sines and cosines - as natural building blocks for constructing the modulation protocols designed to filter time-varying noise over a user defined band while enacting a nontrivial qubit rotation. The Walsh functions are defined in a uniform piecewise-constant fashion, building intrinsic compatibility with discrete clocking [54] and classical digital logic, and have previously been identified as providing a powerful mathematical framework in the context of quantum control sequencing [51]. Moreover, they may be arbitrarily combined using Fourier-like synthesis using techniques for arbitrary waveform generation well established in digital signal processing.

We treat a Walsh-modulated driven qubit system weakly interacting with both dephasing and coherent amplitude-damping noise processes. The task of finding Walshsynthesized modulation patterns that produce effective filters is reduced to minimizing a cost function measuring the extent to which noise over a user-defined spectral band is filtered by the applied control. The performance of resulting control protocols is completely characterized by their Walsh spectra, facilitating intuitive analytic design rules based on symmetry and spectral properties of the Walsh basis. Our work details the various techniques and mathematical constructs through which we define and then optimize the filtersynthesis process in the Walsh basis, and considers the effects of realistic experimental constraints such as limited modulation bandwidth.

With these insights, we derive novel families of Walsh-modulated noise filters designed to suppress dephasing and coherent amplitude-damping noise, and describe their properties. Modulation protocols are tailored to a particular operation on $\mathrm{SU}(2)$, but are other- 
wise largely model-robust (being tailored to suppress noise over a frequency band rather than to a specific time-domain noise signal), and portable between different qubit technologies. Combined with the discovery, presented here, that several prominent composite pulse protocols derived in NMR actually fall within the Walsh-synthesis basis - mirroring similar insights in the context of dynamical decoupling [51] - this work positions the Walsh functions as a natural basis for crafting physical-layer error suppression strategies for scalable quantum technologies.

The remainder of this paper is organized as follows. In Section 2 we describe our model quantum system by defining relevant control and noise Hamiltonians. In Section 3 we review the generalized filter-transfer function formalism used to derive a spectral representation of the operational infidelity. Notation for defining and parameterizing the control space is introduced and explicit expressions for computing corresponding filter functions are presented. Section 4 provides a formal definition of a filter cost function used for optimizing operational fidelity over the control space and deriving useful filters. Performance characteristics of these filters are discussed and interpreted, with care taken to differentiate filter order from Magnus order. In Section 5 physically motivated constraints on the control space are established by synthesizing control waveforms as superpositions of functions in the Walsh basis, bounding the dimensionality of the filter-optimization task. Two useful representations of the Walsh basis - Paley ordering and the Hadamard representation - are introduced. We then develop a range of analytic filter-design rules for efficient filter construction based on the symmetry and spectral properties of the Walsh functions. In Sections 6-9 we apply the above framework to derive several novel families of noise filters implementing nontrivial logic gates. These include filters for dephasing and coherent amplitude-damping noise in addition to concatenated filters for universal noise. In Section 10 we study how relaxing the assumption of perfect square pulses reduces the performance of filters optimized in the Walsh basis, and demonstrate that these filter properties may be recovered in general by simply re-optimizing under the assumption of non-square pulses. We then close with a brief summary and outlook, followed by a number of appendices containing detailed derivations of relevant quantities used in the main text.

\section{Physical setting}

We begin by establishing the Hamiltonian framework for the control and noise interactions treated in this paper. This is necessary background in order to study noise filtering via Walsh-synthesized control fields implementing logic gates. We consider a model quantum system consisting of an ensemble of identically prepared noninteracting qubits immersed in a weakly interacting noise bath and driven by an external control device. Working in the interaction picture with respect to the qubit splitting, state transformations are represented as unitary rotations of the Bloch vector. In this interaction picture the generalized time-dependent Hamiltonian is written

$$
H(t)=H_{c}(t)+H_{0}(t)
$$

where $H_{c}(t)$ describes perfect control of the qubit state, e.g. via an ideal external driving field, and the noise Hamiltonian $H_{0}(t)$ captures undesirable interactions with a timevarying non-Markovian noise environment. The full qubit dynamics are governed by the 
Schrodinger equation $i \dot{U}(t, 0)=H(t) U(t, 0)$ where the time-evolution operator $U(t, 0)$ transforms an initial state $|\psi(0)\rangle$ to the final $U(\tau, 0)|\psi(0)\rangle$ after an interaction of duration $\tau$.

In the absence of noise the total Hamiltonian reduces to $H(t)=H_{c}(t)$, in which case timeevolution is determined purely by control operations according to $i \dot{U}_{c}(t, 0)=H_{c}(t) U_{c}(t, 0)$. An intended evolution path under ideal control is therefore described by the control propagator $U_{c}(t, 0)=\mathcal{T} \exp \left(-i \int_{0}^{t} H_{c}\left(t^{\prime}\right) d t^{\prime}\right)$, with $\mathcal{T}$ denoting the time-ordering operator. For a single qubit the time-dependent control Hamiltonian may in general be written $H_{c}(t)=\Omega(t) \hat{\mathbf{n}}(t) \cdot \boldsymbol{\sigma} / 2$. Here $\hat{\mathbf{n}}(t) \cdot \boldsymbol{\sigma} \equiv n_{x} \hat{\sigma}_{x}+n_{y} \hat{\sigma}_{y}+n_{z} \hat{\sigma}_{z}$ is the rotation generator, $\hat{\mathbf{n}}(t) \in \mathbb{R}$ is a unit vector defining the instantaneous axis of rotation, and $\Omega(t)$ is the instantaneous rate of rotation (Rabi rate) for the Bloch vector.

Environmental interactions are modeled semi-classically, with stochastic noise processes expressed in terms of time-dependent fluctuating classical noise fields. We consider time-dependent dephasing (detuning) and coherent amplitude-damping processes, captured respectively through (stochastic) rotations about $\hat{\sigma}_{z}$ and about the instantaneous direction of control $\hat{\mathbf{n}}(t) \cdot \boldsymbol{\sigma}$. The universal noise Hamiltonian thus takes the form $H_{0}(t)=H_{0}^{(z)}(t)+H_{0}^{(\Omega)}(t)$ where $H_{0}^{(z)}(t)$ and $H_{0}^{(\Omega)}(t)$ denote noise interactions in the dephasing and amplitude noise quadratures respectively. Dephasing noise thus contributes the additive term

$$
H_{0}^{(z)}(t)=\beta_{z}(t) \hat{\sigma}_{z}
$$

where $\beta_{z}(t)$ describes a time-varying noise field. Coherent amplitude-damping noise contributes the multuplicative term

$$
H_{0}^{(\Omega)}(t)=\frac{\beta_{\Omega}(t) \Omega(t)}{2} \hat{\mathbf{n}}(t) \cdot \sigma=\beta_{\Omega}(t) H_{c}(t) .
$$

Including this term is equivalent to making the substitution $\Omega(t) \longrightarrow \Omega(t)\left(1+\beta_{\Omega}(t)\right)$ in the control Hamiltonian, where $\beta_{\Omega}(t)$ describes a (multiplicative) noise source in the amplitude of the driving field. Inclusion of this term in the noise Hamiltonian enables us to go beyond previous studies where attention has been restricted to dephasing processes. This novel approach is important for most realistic experimental situations where correctable non-Markovian amplitude-damping errors arise from noise in the control system itself (for example, fluctuations in the strength of the driving field).

In our model both $\beta_{z}(t)$ and $\beta_{\Omega}(t)$ are assumed to be classical random variables with zero mean and non-Markvovian power spectra. We also assume they are wide sense stationary and independent. ${ }^{\mathrm{a}}$ The former implies the autocorrelation functions $\left\langle\beta_{i}\left(t_{1}\right) \beta_{i}\left(t_{2}\right)\right\rangle$, $i \in\{z, \Omega\}$, depend only on the time difference $t_{1}-t_{2}$. The latter implies the cross-correlation functions vanish. That is, $\left\langle\beta_{i}\left(t_{1}\right) \beta_{j}\left(t_{2}\right)\right\rangle=0$ where $i, j \in\{z, \Omega \mid i \neq j\}$. The angle brackets denote a time average of the random variables. Finally, our model permits access to a wide range of parameter regimes, from quasistatic (noise slow compared to $H_{c}(t)$ ) to the limit in which the noise fluctuates on timescales comparable to or faster than $H_{c}(t)$.

These noise Hamiltonians generate uncontrolled rotations in the qubit dynamics, leading to errors in the evolution path (and hence the final state) relative to the target transformation intended under $H_{c}(t)$. An estimate for this error is derived in the next section using the generalized filter-transfer function formalism. 


\section{Building noise filters}

Overall, our objective is to craft control protocols such that the deleterious effects of timedependent noise on the intended evolution of an arbitrary qubit transformation are suppressed - filtered by the control. Accordingly, we require a measure for the operational fidelity in the presence of both noise and the relevant control. For this we employ the method developed by Green et al. [49]. In this framework the error contributed by the noise fields over the duration of the control is approximated, to first order, via a truncated Magnus expansion. Each noise field then contributes a term to the gate infidelity in the spectral domain expressed as an overlap integral between the noise power spectrum and an appropriate generalized filter-transfer function. We describe this in detail below.

\subsection{Calculating operational fidelity}

In the absence of noise interactions, state evolution is determined by $i \dot{U}_{c}(t)=H_{c}(t) U_{c}(t)$ with $U_{c}(t)$ the ideal evolution operator describing the target operation. Including the effects of noise, however, time evolution is determined by the operator $U(t)$ satisfying $i \dot{U}(t)=\left(H_{c}(t)+H_{0}^{(z)}(t)+H_{0}^{(\Omega)}(t)\right) U(t)$. Our measure for operational fidelity is given by $\mathcal{F}_{a v}(\tau)=\frac{1}{4}\left\langle\left|\operatorname{Tr}\left(U_{c}^{\dagger}(\tau) U(\tau)\right)\right|^{2}\right\rangle$, effectively measuring the extent to which the intended and realized operators 'overlap', as captured by the Hilbert-Schmidt inner product [55]. Computing the evolution dynamics, however, is very challenging since the control and noise Hamiltonians do not commute at different times; sequential application of the resulting time-dependent, non-commuting operations gives rise to both dephasing and depolarization errors, mandating approximation methods.

Our error model assumes non-dissipative qubit evolution with both control and noise interactions resulting in unitary rotations. Hence we approximate the evolution operator as a unitary using a Magnus expansion [56, 57]. This involves moving to a frame corotating with the control known as the toggling frame, originally appearing in the development of average Hamiltonian theory [58]. This approach allows us to separate the part of the system evolution due solely to the control from the part affected by environmental coupling, and is standard in the study of coherent control in NMR $[58,59]$ and quantum information.

Defining the error propagator $\tilde{U}(t) \equiv U_{c}^{\dagger}(t) U(t)$, the total evolution operator is written $U(t)=U_{c}(t) \tilde{U}(t)$. In this case the realized evolution operator approaches the target operation as $\tilde{U}(\tau) \rightarrow \mathbf{I}$, establishing the condition for suppression of noisy evolution dynamics. However, moving to the toggling frame defined by toggling frame Hamiltonian $\tilde{H}_{0}(t) \equiv U_{c}^{\dagger}(t) H_{0}(t) U_{c}(t)$, the error propagator satisfies the Schrodinger equation $i \dot{\tilde{U}}(t)=\tilde{H}_{0}(t) \tilde{U}(t)$. Performing a Magnus expansion in this frame - assuming convergence of the series [57] - we may write $\tilde{U}(\tau)=\exp \left[-i \sum_{\mu=1}^{\infty} \mathbf{a}_{\mu}(\tau) \cdot \boldsymbol{\sigma}\right]$ where the error vectors $\mathbf{a}_{\mu}(\tau)$ determine expansion coefficients of the Magnus series operators $\Phi_{\mu}(\tau)$ expressed in the basis of Pauli matrices (see Appendix A). We may then in principle approximate $\tilde{U}(t)$ to arbitrary accuracy by truncating the infinite series at an appropriate order.

The operational fidelity $\mathcal{F}_{a v}(\tau)=\frac{1}{4}\left\langle|\operatorname{Tr}(\tilde{U}(\tau))|^{2}\right\rangle$ may now be fully expressed as an infinite power series over the ensemble-averaged magnitudes of the expansion vectors $\mathbf{a}_{\mu}(\tau)$. In the limit of sufficiently weak noise, ${ }^{\mathrm{b}}$ however, it is appropriate to truncate the expansion to first-order yielding $\mathcal{F}_{a v}(\tau) \approx 1-\left\langle a_{1}^{2}\right\rangle$ with $\left\langle a_{1}^{2}\right\rangle \equiv\left\langle\mathbf{a}_{1}(\tau) \mathbf{a}_{1}^{T}(\tau)\right\rangle$ defining the first order infidelity. Now, as set out in Appendix A the first-order error vector is related to the first-order Magnus term according to Eq. (70), yielding $\mathbf{a}_{1}(\tau) \cdot \sigma=\Phi_{1}(\tau)=\int_{0}^{\tau} d t \tilde{H}_{0}(t)$. 
That is, the first-order infidelity $\left\langle a_{1}^{2}\right\rangle$ is associated with the time-average of the toggling frame Hamiltonian over the total sequence duration.

Expressing $\tilde{H}_{0}(t) \equiv \overrightarrow{\mathcal{R}}(t) \cdot \boldsymbol{\sigma}$ in the Pauli basis, where the expansion vector $\overrightarrow{\mathcal{R}}(t)$ is some convolution of both control and noise fields, we obtain the computational expression $\mathbf{a}_{1}(\tau)=\int_{0}^{\tau} d t \overrightarrow{\mathcal{R}}(t)$. Using the noise model assumptions outlined in Section 2, and performing a number of Fourier-like transforms (see Appendix A for full details), we obtain a spectral representation of the form

$$
\left\langle a_{1}^{2}\right\rangle=\frac{1}{2 \pi} \int_{-\infty}^{\infty} \frac{d \omega}{\omega^{2}} S_{z}(\omega) F_{z}(\omega)+\frac{1}{2 \pi} \int_{-\infty}^{\infty} \frac{d \omega^{\prime}}{\omega^{\prime 2}} S_{\Omega}\left(\omega^{\prime}\right) F_{\Omega}\left(\omega^{\prime}\right) .
$$

Here $S_{z}(\omega)$ and $S_{\Omega}(\omega)$ denote the dephasing and amplitude noise power-spectral densities (PSDs). The dephasing $F_{z}(\omega)$ and amplitude $F_{\Omega}(\omega)$ filter-transfer functions, on the other hand, capture the spectral response of the control sequence. Moving forward, we will present the mathematical framework that permits calculation of these quantities for arbitrary control protocols.

\subsection{Defining the control space}

In order to realize specific noise filters, characterized by the filter-transfer functions introduced above, we require a simple framework to define the time-domain control operations that can be applied to the qubit. Representing the qubit state on the Bloch sphere, state manipulation maps to a rotation in $\mathbb{R}^{3}$ of the Bloch vector associated with a unitary transformation $\mathcal{U}\left(\theta, \hat{\sigma}_{\hat{\mathbf{n}}}\right) \equiv \exp [-i(\boldsymbol{\sigma} \cdot \hat{\mathbf{n}}) \theta / 2]$, reflecting the homeomorphism between $\operatorname{SU}(2)$ and $S O(3)$. The rotation generator $\hat{\sigma}_{\hat{\mathbf{n}}} \equiv \hat{\mathbf{n}} \cdot \boldsymbol{\sigma} \equiv n_{x} \hat{\sigma}_{x}+n_{y} \hat{\sigma}_{y}+n_{z} \hat{\sigma}_{z}$ produces a rotation though an angle $\theta$ about the axis defined by the unit vector $\hat{\mathbf{n}} \in \mathbb{R}^{3}$.

We treat control protocols taking the form of an $n$-segment sequence of such unitaries, executed over the time period $[0, \tau]$. This implies a natural partition of the total sequence duration $\tau$ into $n$ subintervals $I_{l}=\left[t_{l-1}, t_{l}\right], l \in\{1, \ldots, n\}$, such that the $l$ th control unitary has duration $\tau_{l}=t_{l}-t_{l-1}$. Here $t_{l-1}$ and $t_{l}$ are the start and end times of the $l$ th rotation respectively, and we define $t_{0} \equiv 0$ and $t_{n} \equiv \tau$. In particular we consider control unitaries of the form

$$
\begin{aligned}
& P_{l} \equiv \mathcal{U}\left(\Omega_{l} \tau_{l}, \hat{\sigma}_{\phi_{l}}\right)=\exp \left[-i \frac{\Omega_{l}}{2} \tau_{l} \hat{\sigma}_{\phi_{l}}\right], \\
& \hat{\sigma}_{\phi_{l}} \equiv \cos \left(\phi_{l}\right) \hat{\sigma}_{x}+\sin \left(\phi_{l}\right) \hat{\sigma}_{y},
\end{aligned}
$$

corresponding to the experimentally relevant case of a resonantly driven qubit. Here $\Omega_{l}$ is the Rabi rate during the $l$ th time interval $\left[t_{l-1}, t_{l}\right]$, and is assumed constant over the duration $\tau_{l}$ of the associated control interaction. During this interaction the rotation generator $\hat{\sigma}_{\phi_{l}}$, parameterized by $\phi_{l} \in[0,2 \pi]$, thus generates a rotation of the Bloch vector through an angle $\theta_{l} \equiv \Omega_{l} \tau_{l}$ about the axis $\hat{\mathbf{n}}_{l} \equiv\left(\cos \left(\phi_{l}\right), \sin \left(\phi_{l}\right), 0\right)$ in the $x y$ plane. $^{\mathrm{c}}$ The control Hamiltonian associated with this $n$-segment sequence takes the form

$$
H_{c}(t)=\sum_{l=1}^{n} G^{(l)}(t) \frac{\Omega_{l}}{2} \hat{\sigma}_{\phi_{l}},
$$


where the function $G^{(l)}(t)$ is 1 if $t \in I_{l}$ and zero otherwise. Controlled evolution during the $l$ th time interval is, under this Hamiltonian, consequently described by the unitary

$$
U_{c}\left(t, t_{l-1}\right)=\exp \left[-i \frac{\Omega_{l}}{2} \hat{\sigma}_{\phi_{l}}\left(t-t_{l-1}\right)\right]
$$

That is, implementation of the $l$ th completed rotation is equivalently denoted by the operator $P_{l}=U_{c}\left(t_{l}, t_{l-1}\right)$. For compactness we define the cumulative operator

$$
Q_{l}:=P_{l} P_{l-1} \cdots P_{0}, \quad P_{0}:=\mathbf{I}
$$

to capture the cumulative action of the first $l$ sequentially competed rotations. Hence the control propagator at any time $t$ may be written

$$
U_{c}(t, 0)=\sum_{l=1}^{n} G^{(l)}(t) U_{c}\left(t, t_{l-1}\right) Q_{l-1}
$$

$H_{c}(t)$ is thus completely described by the sequence of $n$ triples $\left\{\left(\Omega_{l}, \tau_{l}, \phi_{l}\right)\right\}_{l=1}^{n}$, and each control operation is completely parameterized by the control variables according to $P_{l}=$ $P_{l}\left(\theta_{l}, \Omega_{l}, \tau_{l}, \phi_{l}\right)$. Although not strictly an independent parameter it is useful to include $\theta_{l}=\Omega_{l} \tau_{l}$ in the argument to distinguish different realizations of the same net rotation for different choices of $\Omega_{l} \tau_{l}$. We define the $(n \times 4) n$-segment matrix

$$
\boldsymbol{\Gamma}_{n}=\begin{gathered}
P_{1} \\
\vdots \\
P_{n}
\end{gathered}\left[\begin{array}{cccc}
\Omega_{l} & \tau_{l} & \theta_{l} & \phi_{l} \\
\Omega_{1} & \tau_{1} & \theta_{1} & \phi_{1} \\
\Omega_{2} & \tau_{2} & \theta_{2} & \phi_{2} \\
\vdots & \vdots & \vdots & \vdots \\
\Omega_{n} & \tau_{n} & \theta_{n} & \phi_{n}
\end{array}\right]
$$

to compactly describe any arbitrary $n$-segment unitary control sequence (see Figure 1 ). The entire space $\mathfrak{C}_{n}$ of such control forms, referred to the $n$-segment control space, and written formally

$$
\mathfrak{C}_{n}:=\left\{\boldsymbol{\Gamma}_{n} \mid \Omega_{l}, \theta_{l}, \tau_{l}>0, \phi_{l} \in[0,2 \pi], l \in 1, \ldots, n, \sum_{l}^{n} \tau_{l}=\tau\right\}
$$

thus corresponds to an infinite set of $\boldsymbol{\Gamma}_{n}$ matrices ranging continously over all possible values taken by the control variables. This general class of control, consisting of boundedstrength unitary sequences, includes familiar composite-pulse sequences in NMR and DCGs in quantum information. We use the more general control space, however, to construct novel qubit gates specifically designed to filter non-Markovian noise.

\subsection{Generalized filter-transfer functions}

We now present the computational forms of the filter-transfer functions $F_{z}(\omega)$ and $F_{\Omega}(\omega)$ introduced in Eq. (4) for arbitrary $n$-segment control protocols implemented by Eq. (7). As outlined above, the filter-transfer functions are completely parametrized by the control 
Figure 1 Filter construction. Visualization of the available control space for an $n=8$ segment control sequence. The filter is synthesized over the parameters presented in $\boldsymbol{\Gamma}_{n}$, whose transpose corresponds to the discrete time segments in the time-domain filter. As an illustration, a time-varying Rabi rate (arbitrary units) is presented for each of the I segments. Synthesis of this waveform may be constructed in the Walsh basis using the Hadamard transform (notation upper right), as will be discussed in Section 5.

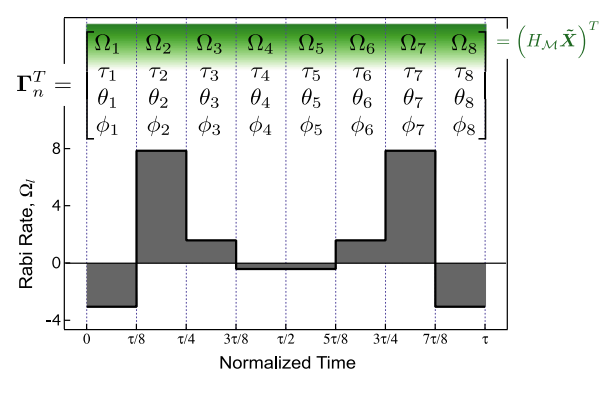

variables $\left\{\left(\Omega_{l}, \tau_{l}, \phi_{l}\right)\right\}_{l=1}^{n} \cong \boldsymbol{\Gamma}_{n}$. Here we only provide a summary of the relevant computational quantities, leaving the major derivations and full explanation to Appendix A. We start by writing

$$
\begin{aligned}
& F_{z}(\omega):=\left[\mathbf{R}^{(z)}(\omega)\right]^{*}\left[\mathbf{R}^{(z)}(\omega)\right]^{T} \quad \text { (Dephasing Filter-Transfer Function), } \\
& F_{\Omega}(\omega):=\left[\mathbf{R}^{(\Omega)}(\omega)\right]^{*}\left[\mathbf{R}^{(\Omega)}(\omega)\right]^{T} \quad \text { (Amplitude Filter-Transfer Function) },
\end{aligned}
$$

where the row vectors $\mathbf{R}^{(z)}(\omega), \mathbf{R}^{(\Omega)}(\omega) \in \mathbb{R}^{3}$ are obtained by Fourier transforming relevant time-domain functions associated with the control evolution dynamics. In Appendix B we derive the explicit computational forms

$$
\begin{aligned}
& \left.\mathbf{R}^{(z)}(\omega)=\sum_{l=1}^{n} e^{i \omega t_{l-1}} \mathbf{R}_{z}^{P_{l}}(\omega) \mathbf{\Lambda}^{(l-1)} \quad \text { (Dephasing Control Vector }\right), \\
& \mathbf{R}^{(\Omega)}(\omega)=\sum_{l=1}^{n}\left[e^{i \omega t_{l-1}}-e^{i \omega t_{l}}\right] \overrightarrow{\mathbb{T}}^{(l)} \Lambda^{(l-1)} \quad \text { (Amplitude Control Vector). }
\end{aligned}
$$

The row vector $\mathbf{R}_{z}^{P_{l}}(\omega) \in \mathbb{R}^{3}$ captures the spectral response in the dephasing noise quadrature contributed during the $l$ th unitary control segment. This takes the form (see Appendix B)

$$
\mathbf{R}_{z}^{P_{l}}(\omega)=\frac{\omega}{\omega^{2}-\Omega_{l}^{2}}\left(\begin{array}{c}
\sin \left(\phi_{l}\right)\left[i \Omega_{l} e^{i \omega \tau_{l}} \cos \left(\Omega_{l} \tau_{l}\right)+\omega e^{i \omega \tau_{l}} \sin \left(\Omega_{l} \tau_{l}\right)-i \Omega_{l}\right] \\
-\cos \left(\phi_{l}\right)\left[i \Omega_{l} e^{i \omega \tau_{l}} \cos \left(\Omega_{l} \tau_{l}\right)+\omega e^{i \omega \tau_{l}} \sin \left(\Omega_{l} \tau_{l}\right)-i \Omega_{l}\right] \\
i \Omega_{l} e^{i \omega \tau_{l}} \sin \left(\Omega_{l} \tau_{l}\right)-\omega e^{i \omega \tau_{l}} \cos \left(\Omega_{l} \tau_{l}\right)+\omega
\end{array}\right)^{T} .
$$

We also define the lth-Segment Projection Vector

$$
\overrightarrow{\mathbb{T}}^{(l)}:=\frac{\Omega_{l}}{2}\left(\cos \left(\phi_{l}\right), \sin \left(\phi_{l}\right), 0\right)
$$

to compactly express the control variables - namely Rabi rate and the rotation-axis vector, projected onto the $x y$ plane of the Bloch sphere - associated with evolution during the $l$ th unitary. In fact, inspection of Eqs. (15) and (16) reveals that $\overrightarrow{\mathbb{T}}^{(l)}$ is the computational analogue of $\mathbf{R}_{z}^{P_{l}}$ for the amplitude noise quadrature. The simpler dependence of $\vec{T}^{(l)}$ on the control variables, however, reflects the fact that amplitude noise in our model is always coaxial, and hence commutes with, the control. 
On the other hand, the $3 \times 3$ Control History Matrix $\mathbf{\Lambda}^{(l-1)}$, defined by

$$
\Lambda_{i j}^{(l-1)}=\frac{1}{2} \operatorname{Tr}\left[Q_{l-1}^{\dagger} \hat{\sigma}_{i} Q_{l-1} \hat{\sigma}_{j}\right]
$$

is the result of expanding the operator $Q_{l-1}^{\dagger} \hat{\sigma}_{i} Q_{l-1} \equiv \sum_{j} \Lambda_{i j}^{(l-1)} \hat{\sigma}_{j}$, with $i, j \in\{x, y, z\}$, in the Pauli basis, and identifying the coefficients $\Lambda_{i j}^{(l-1)}$ as the matrix elements. $\Lambda^{(l-1)}$ thereby captures the accumulated effect of the previous $l-1$ completed unitaries, implemented via the cumulative operator $Q_{l-1}$.

\section{Characteristics of noise filters}

The power of the noise filtering formalism lies in the simple interpretation of the filtertransfer functions $F_{i}(\omega)$, which may be characterized in a standard engineering approach, considering passbands, stopbands, and filter order $[42,48,49,60]$. In particular, error suppression corresponds to minimizing $F_{i}(\omega), i \in\{z, \Omega\}$ in the spectral region where the corresponding PSDs are non-negligible. This can, in principle, be achieved by judicious construction of the control sequence since the filter-transfer functions are completely parametrized in variables describing the time-domain control applied to the qubit.

We are now in a position to examine the characteristics of the filter-transfer functions for an arbitrary control sequence $\boldsymbol{\Gamma}_{n}$, formally indicating the functional dependence of the filter-transfer functions on the control variables by writing $F_{i}(\omega \tau)=F_{i}\left(\omega \tau ; \boldsymbol{\Gamma}_{n}\right), i \in$ $\{z, \Omega\}$. Inversely, we may commence a study of filter design based on constructing control sequences satisfying some desired filter property - our main goal. We now advance the main mathematical framework used in this paper to study filter design, pulling together the ideas introduced in the previous sections.

\subsection{The filter cost function}

A definition of the cost function associated with filter performance - captured through the filter order - leads us naturally to the imposition of constraints on the available space of controls. This cost function therefore lies at the heart of our attempts to craft control protocols appropriate for a given noise environment.

From the spectral overlap in Eq. (4), minimizing the infidelity contributed by the noise process $S_{i}(\omega)$ corresponds to minimizing the area under $F_{i}\left(\omega \tau ; \boldsymbol{\Gamma}_{n}\right)$ in the spectral region of interest. We therefore define a cost function over a user-defined frequency band taking the form

$$
A_{i}\left(\boldsymbol{\Gamma}_{n}\right):=\int_{\omega_{L}}^{\omega_{c}} d \omega F_{i}\left(\omega \tau ; \boldsymbol{\Gamma}_{n}\right), \quad i \in\{z, \Omega\}
$$

to diagnose the filtering effectiveness achieved by the control sequence $\boldsymbol{\Gamma}_{n}$. The smaller the integral $A_{i}\left(\boldsymbol{\Gamma}_{n}\right)$, the more effective the noise filtering over this band, in this noise quadrature. Since $\boldsymbol{\Gamma}_{n}$ is defined continuously over $\mathfrak{C}_{n}$ for a given $n$, we may in principle construct a variational procedure over this control space to derive minimizing 'values' of $\boldsymbol{\Gamma}_{n}$ satisfying a given cost function. In effect, the problem involves solving for paths in the control space over which the functional $A_{i}\left(\boldsymbol{\Gamma}_{n}\right)$ is minimized (up to some order).

Typically one would define the band $\left[\omega_{L}, \omega_{c}\right]$ over which the cost function is defined to fall within the stopband of $F_{i}(\omega \tau)$, below which filtering generally takes place. In general 
the band $\left[\omega_{L}, \omega_{c}\right]$ may be tailored to target specific spectral regions in the noise PSD. Doing so may produce highly effective filtering over this narrow spectral region, though out-ofband behaviour can be quite poor if not specifically optimized. ${ }^{\mathrm{d}}$

\subsection{The filter order}

Again, following concepts from filtering in classical control engineering, we may define a filter order which will play a central role in efficiently realizing effective noise filters. We will mainly consider high-pass filters for low-frequency noise, setting $\omega_{L}=0$ such that filtering takes place in the stopband up to the cutoff $\omega_{c}$. In this case it is useful to perform the Taylor expansion of the filter-transfer function about $\omega=0$, written

$$
F_{i}\left(\omega \tau ; \boldsymbol{\Gamma}_{n}\right)=\sum_{k=1}^{\infty} C_{2 k}^{(i)}\left(\boldsymbol{\Gamma}_{n}\right)(\omega \tau)^{2 k}
$$

where the dependence of the expansion coefficients $C_{2 k}^{(i)}$ on $\Gamma_{n}$ has been made explicit, and we include only even powers of $\omega \tau$ due to the evenness of $F_{i}(\omega \tau)$. Assuming sufficiently low-frequency noise $\left(\omega_{c}<1 / \tau\right)$, the approximation $F(\omega \tau) \propto(\omega \tau)^{2 p}$ holds for some $p$ associated with the most significant power law expansion term. This defines a high-pass filter with filter order (determined by $p$ ) visualized as the slope in the stopband on a log-log plot. $^{\text {e }}$

Using this notation, and working in the low-frequency limit, we then say the control sequence $\boldsymbol{\Gamma}_{n} \in \mathfrak{C}_{n}$ filters $\beta_{i}(t)$ noise to order $(p-1)$ over the band [0, $\left.\omega_{c}\right]$ if $\boldsymbol{\Gamma}_{n}$ is a concurrent zero of the first $(p-1)$ Taylor coefficients. That is, if $C_{2}^{(i)}\left(\boldsymbol{\Gamma}_{n}\right)=C_{4}^{(i)}\left(\boldsymbol{\Gamma}_{n}\right)=\cdots=$ $C_{2(p-1)}^{(i)}\left(\boldsymbol{\Gamma}_{n}\right)=0$. In this case we approximate $F_{i}\left(\omega \tau ; \boldsymbol{\Gamma}_{n}\right) \approx C_{2 p}^{(i)}\left(\boldsymbol{\Gamma}_{n}\right)(\omega \tau)^{2 p}$ and consequently $A_{i}\left(\boldsymbol{\Gamma}_{n}\right) \approx C_{2 p}^{(i)}\left(\boldsymbol{\Gamma}_{n}\right) \frac{\left(\omega_{c} \tau\right)^{2 p+1}}{2 p+1}$. Thus $\boldsymbol{\Gamma}_{n}$ is a $(p-1)$-order (high-pass) filter in the $i$ th noise quadrature if the following equivalent conditions are satisfied

$$
\frac{A_{i}\left(\boldsymbol{\Gamma}_{n}\right)}{C_{2 p}^{(i)}\left(\boldsymbol{\Gamma}_{n}\right)}=\mathcal{O}\left(\frac{\left(\omega_{c} \tau\right)^{2 p+1}}{2 p+1}\right) \quad \Longleftrightarrow \quad C_{2}^{(i)}\left(\boldsymbol{\Gamma}_{n}\right)=\cdots=C_{2(p-1)}^{(i)}\left(\boldsymbol{\Gamma}_{n}\right)=0 .
$$

This metric will play a central role in the analyses that follow.

It is important to disambiguate the asymptotic filter order $(p-1)$, introduced above for characterizing the behaviour near zero frequency, from a more general metric capable of describing filter performance over an arbitrary spectral band. For this we introduce the local filter order $\left(p^{*}-1\right)$ by the property that, over the band $\left[\omega_{L}, \omega_{c}\right]$ the filter-transfer function is well approximated by $F_{i} \propto(\omega \tau)^{2 p^{*}}$. One may take the limit that $\omega_{L} \rightarrow \omega_{c} \rightarrow \omega^{*}$ and thereby obtain the instantaneous filter order, effectively measuring the power-law behaviour at $\omega^{*}$. Both local and instantaneous filter order reduce to the asymptotic filter order over the stopband if over this region $F_{i}$ is well-characterized by its the zero-frequency behaviour.

\subsection{Time-domain filter order vs. Magnus order}

Both the asymptotic and instantaneous filter orders defined above for time-domain noise must be distinguished from the Magnus order of error cancellation. The latter is familiar from work in NMR in which quasi-static errors can be cancelled by suitable composite pulse sequence design. The regime of quasistatic errors coincides with the DC limit 
for the time-dependent noise fields introduced in Section 2. That is, the time-dependent noise fields reduce to scalar constants $\beta_{z}\left(\beta_{\Omega}\right)$. The Magnus expansion terms in (70), now denoted $\Phi_{\mu}^{(\mathrm{DC})}$, are then evaluated strictly as time integrals over ideal control operations, scaled by factors $\beta_{z}^{\mu}\left(\beta_{\Omega}^{\mu}\right)$ specifying the power law dependence on the magnitude of these static offsets errors. A pulse sequence for which $\Phi_{1}^{(\mathrm{DC})}=\cdots=\Phi_{\mu-1}^{(\mathrm{DC})}=0$ is then said to compensate offset errors to Magnus order $(\mu-1)$. In this case the total error operator satisfies $\Phi^{(\mathrm{DC})}(\tau)=\mathcal{O}\left(\Phi_{\mu}^{(\mathrm{DC})}\right)$ and is dominated by the residual error proportional to the $\mu$ th power in the magnitude of the error.

This is quite distinct from time-dependent noise where the error expansion used to calculate the fidelity contains terms of various Magnus order but equivalent time-dependent error norm in the ensemble average (see, e.g. Eq. 1 in Ref. [48]). The net result is the observation that high-order error suppression in the Magnus expansion does not imply highorder time-domain noise filtering. This has been validated using experiments on trapped ions [37], and formalized rigorously in Ref. [38], where it has been shown that $p \leq \mu$, but $p^{*}$ over a user-defined band is unrelated to $\mu$. Our focus throughout this work will be on crafting efficient noise filters rather than high-order error suppressing gates.

\section{Filter design by Walsh synthesis}

Even with the general insights into the appropriate modulation protocols outlined above, it is desirable to bound the dimensionality of the control space, and hence the complexity of the filter-design task, by imposing physically motivated constraints on the form of $\boldsymbol{\Gamma}_{n}$. In practice the achievable filter order is typically limited by the number of unitary operations in the control sequence; one may increase $(p-1)$ at the cost of increasing $n$. From an experimental standpoint, faced with the physical limitation set by a maximum achievable Rabi rate, this cost manifests as a longer total sequence duration $\tau=\hat{\sigma}_{l}^{n} \tau$. This may offset the proposed benefit of the higher-order filter due to a longer noise interaction time. From a theoretical standpoint the cost is in the greater complexity of the variational search; the number of (free) variational parameters in $\boldsymbol{\Gamma}_{n}$ grow as $3 n$ and the number of matrix products in Eqs. (15) and (16) grows as $n$.

We are able to effectively bound the synthesis space while still achieving highly effective gates by synthesizing relevant time-domain control fields in the basis set of Walsh functions - square wave analogues of the sines and cosines [51, 61] - using the concept of functional analysis. Walsh functions are defined in a uniform piecewise-constant fashion (Figure 2), building intrinsic compatibility with discrete clocking [54] and classical digital logic. Since their formulation in the first half of the twentieth century [62] they have played an important role in scientific and engineering applications. Their development and utilization has been strongly influenced by parallel developments in digital electronics and computer science since the 1960s, with Walsh-type transforms replacing Fourier transforms in a range of engineering applications such as communication, signal processing, pattern recognition, noise filtering and so forth [61, 63].

More recently the Walsh functions have been identified as an attractive resource in quantum information, with applications in time-resolved magnetometry using nitrogenvacancy centres in diamond [64] and in DD for digital-efficient pulse sequencing [51]. Notably, in the latter scheme the decoupling performance was found to be determined by the distinct symmetry and spectral properties of the Walsh basis. These properties enable us to establish analytic design rules (see Section 5.3) to further streamline Walsh-synthesized filter construction. 
Figure 2 Walsh functions. The first 32

Paley-ordered Walsh functions $P A L_{k}, k \in\{0, \ldots, 31\}$.

Functions with maximum Hamming weight $r(k)$

(hence maximum number of Rademacher

functions) for given $m(k)$, corresponding to Paley

orders $2^{m(k)}-1$, are highlighted in red.

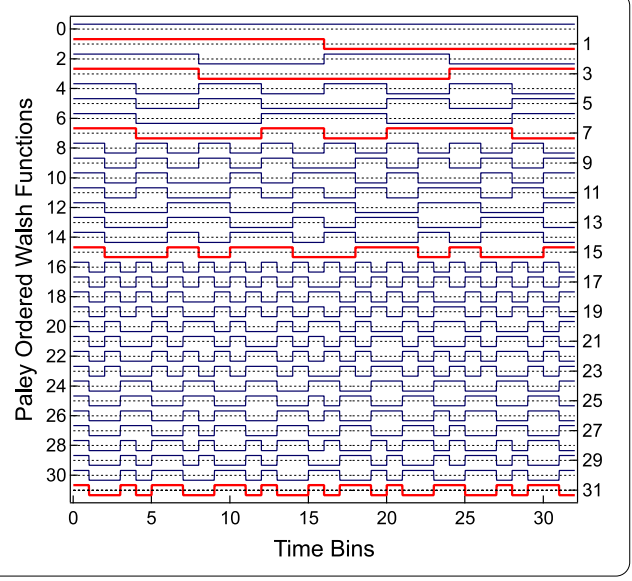

We begin by reviewing the relevant mathematical details of the Walsh basis. Two equivalent representations are introduced, Paley ordering and the Hadamard representation, which shall be used throughout this paper.

\subsection{The Paley and Hadamard representations}

The set of Walsh functions $w_{k}:[0,1] \rightarrow\{ \pm 1\}, k \in \mathbb{N}$ form an orthonormal-complete family of binary-valued square waves defined on the unit interval. They are aperiodic and hence do not admit to a unique ordering, in contrast with the Fourier basis in which sinusoids are ordered by increasing frequency. A number of different orderings exist [61, 65, 66] due to the different ways in which the basis elements may be defined. We employ the Paley ordering [67] in which basis functions are generated from products of Rademacher functions [68], defined by

$$
R_{j}(x):=\operatorname{sgn}\left[\sin \left(2^{j} \pi x\right)\right], \quad x \in[0,1], j \geq 0 .
$$

The $j$ th Rademacher function $R_{j}(x)$ is thus a periodic square wave switching $2^{j-1}$ times between \pm 1 over the interval $[0,1]$. The Walsh function of Paley order $k$, here denoted $\operatorname{PAL}_{k}(x)$, is then defined by

$$
\operatorname{PAL}_{k}(x)=\prod_{j=1}^{m} R_{j}(x)^{b_{j}},
$$

where $\left(b_{m}, b_{m-1}, \ldots, b_{1}\right)_{2}$ is the binary representation of $k$. That is, $k=b_{m} 2^{m-1}+b_{m-1} 2^{m-2}+$ $\cdots+b_{1} 2^{0}$, where $m(k)$ indexes the most significant binary digit, having defined $b_{m} \equiv 1$. Consequently, $\operatorname{PAL}_{k}(x)$ has factors $R_{j}(x)$ whenever $b_{j}$ is a nonzero binary digit of $k$; the total number of Rademacher functions in the construction of $\operatorname{PAL}_{k}(x)$ is thus given by the number of nonzero $b_{j}$ 's in $k$ - namely, the Hamming weight denoted $r(k)$. For a given value of $m(k)$, the maximum number Rademacher functions therefore occur for $\operatorname{PAL}_{2^{m}(k)-1}(x)$. For example, setting $m(k)=3$, a maximum of three Rademacher functions are used to construct $\mathrm{PAL}_{7}(x)=R_{3}(x) R_{2}(x) R_{1}(x)$, corresponding to the three nonzero digits $b_{1,2,3}$ in the binary expansion $k=7=(1,1,1)_{2}$. For illustration, the first 32 Walsh functions in the Paley ordering are shown in Figure 2. 
The discrete-timestep properties of these basis functions produce, under linear superposition, piecewise-constant waveforms with digitized segment lengths. In our framework these segments are used to specify the a modulation of the control field, ultimately defining a piecewise-constant sequence of unitaries. We therefore require a straightforward expression for the envelope of an arbitrary synthesis $\sum_{k=0}^{N} X_{k} \operatorname{PAL}_{k}(x)$. Due to the aperiodicity of the Walsh functions, however, a general expression in Paley ordering is difficult. To overcome this it is convenient to use the Hadamard representation.

The unique sign-switching envelope of $\operatorname{PAL}_{k}(x)$ is determined by the sign-switching of the constituent Rademacher functions. Since any $R_{j}(x)$ switches sign uniformly $2^{j}$ times over the interval $[0,1]$, the fastest sign-modulation rate in $\operatorname{PAL}_{k}(x)$ derives from the highest order Rademacher function $R_{m(k)}(x)$, which switches sign $2^{m(k)}$ times over $[0,1]$. Provided $m(k) \leq n$, we may therefore partition $[0,1]$ into $2^{n}$ equal time bins such that $\operatorname{PAL}_{k}(x)$ is constant valued on each bin. Any basis function $\operatorname{PAL}_{k}(x)$ then projects completely onto a digital vector in $\mathbb{R}^{2^{n}}$ with the $j$ th element, $P_{j}^{(k)} \in\{ \pm 1\}$, defined by the value of $\operatorname{PAL}_{k}(x)$ in the $j$ th bin. That is, $\operatorname{PAL}_{k}(x)$ is isomorphic to the discrete digital vector written

$$
\mathbf{P}_{2^{n}}^{(k)} \equiv\left[\begin{array}{llll}
P_{1}^{(k)}, & P_{2}^{(k)}, & \ldots, & P_{2^{n}}^{(k)}
\end{array}\right]
$$

This projection is possible for all $k \in\left\{0,1, \ldots, 2^{n}-1\right\}$ for which the condition $m(k) \leq n$ is true, resulting in a set of $2^{n}$ vectors. Since these vectors inherit the orthogonality of the $\operatorname{PAL}_{k}(x)$, moreover, they form a discrete Walsh basis spanning $\mathbb{R}^{2^{n}}$.

In the Hadamard representation, these vectors occur as columns (rows) of the Hadamard matrix of dimension $2^{n}$. Using so-called Sylvester construction [69] the $2^{n}$-dimensional Hadamard matrix $H_{2^{n}}$ is generated recursively by

$$
H_{2^{n}}=\left[\begin{array}{cc}
H_{2^{n-1}} & H_{2^{n-1}} \\
H_{2^{n-1}} & -H_{2^{n-1}}
\end{array}\right]=S^{\otimes n}, \quad S=\left[\begin{array}{cc}
1 & 1 \\
1 & -1
\end{array}\right], \quad H_{1}=1,
$$

where $S$ is the Sylvester matrix, and $\otimes n$ denotes $n \geq 1$ applications of the Kronecker product. In this construction $\mathbf{P}_{2^{n}}^{(k)}$ defines the $i(k)=1+\sum_{j=1}^{m(k)} b_{j} 2^{n-j}$ column (row) of $H_{2^{n}}$. The orthogonality of the Walsh basis is thereby reflected in the familiar property that $H_{2^{n}} H_{2^{n}}^{T}=2^{n} I$, implying the orthogonality of the Hadamard matrices.

The Hadamard representation of the Walsh functions has the distinct advantage of naturally specifying the piecewise-constant structure of time domain sequences constructed via linear combinations of Walsh functions. Any function synthesized in the Paley-ordered Walsh basis, $f(x)=\sum_{k=0}^{2^{n}-1} q_{k} \mathrm{PAL}_{k}(x)$, has a vector representation in the column space of $H^{2^{n}}$. In this section we will use this observation to efficiently construct Walsh-synthesized filters, whose properties map compactly onto the Walsh spectrum.

\subsection{Walsh-synthesized filters}

The basic physics of commutation relations between Pauli operators immediately suggests an immediate constraint on the available modulation, broadly involving structuring of the Rabi rate or control phase in the time domain

$$
\begin{aligned}
& \Gamma_{n}^{\mathrm{AMF}} \cong\left\{\left(\tau_{l}, \Omega_{l}\right)\right\}_{l=1}^{n}, \quad \phi_{l}=\phi_{0} \forall l \in\{1, \ldots, n\}, \\
& \Gamma_{n}^{\mathrm{PMF}} \cong\left\{\left(\tau_{l}, \phi_{l}\right)\right\}_{l=1}^{n}, \quad \Omega_{l}=\Omega_{0} \forall l \in\{1, \ldots, n\}
\end{aligned}
$$


referred to as single-axis amplitude-modulated filters (AMFs) and constant-amplitude phase-modulated filters (PMFs). These constrained forms may be used to design filters for dephasing and amplitude noise separately using minimal control resources. For $\hat{\sigma}_{z}$ (dephasing) noise it is sufficient to employ rotations about a single (orthogonal) axis in the $x y$ plane and therefore restrict attention to AMFs. On the other hand, unless implementing the trivial identity gate such that the total gate rotation angle $\Theta \equiv \sum_{l=1}^{\mathcal{M}} \theta_{l}=0$, strict amplitude modulation is insufficient for filtering amplitude noise. ${ }^{\mathrm{f}}$ For nontrivial gates, amplitude noise filters generally require control over the rotation axis, and for this purpose we employ PMFs.

In the Walsh synthesis framework, the modulated structures $\boldsymbol{\Gamma}_{n}^{\mathrm{AMF}}$ and $\boldsymbol{\Gamma}_{n}^{\mathrm{PMF}}$ are further constrained by synthesizing the time-domain Rabi rate $\Omega(t)$ or phase $\phi(t)$ as linear superpositions of Walsh functions

$$
\Omega(t)=\sum_{k=0}^{N} X_{k} \operatorname{PAL}_{k}(t / \tau), \quad \phi(t)=\sum_{k=0}^{N} Y_{k} \operatorname{PAL}_{k}(t / \tau), \quad t \in[0, \tau] .
$$

Here the synthesis spectra are denoted in terms of $X_{k}\left(Y_{k}\right)$ to distinguish Walsh modulation in the amplitude (phase) quadratures. We refer to the resulting sequences as Walsh amplitude- (WAMF) and phase- (WPMF) modulated filters. To compactly express these modulated control forms as sequences of unitaries we now employ the Hadamard representation.

Consider an arbitrary function $f(x)=\sum_{k=0}^{N} q_{k} \operatorname{PAL}_{k}(x)$ synthesized in the Walsh basis up to Paley order N. From Section 5.1 all basis functions in this synthesis projected onto a Hadamard matrix of (minimum) dimension $\mathcal{M}(N) \equiv 2^{m(N)}$. A discrete representation of the function $f(x)$ therefore exists as a projection onto the column space of $H_{\mathcal{M}}$ by writing

$$
\mathbf{f}=H_{\mathcal{M}} \tilde{\mathbf{q}}, \quad \tilde{\mathbf{q}}=\left(\tilde{q}_{1}, \tilde{q}_{2}, \ldots, \tilde{q}_{\mathcal{M}}\right)^{T}, \tilde{q}_{i(k)}= \begin{cases}q_{k} & \text { for } 0 \leq k \leq N \\ 0 & \text { for } N<k<\mathcal{M}\end{cases}
$$

where the column vector $\tilde{\mathbf{q}}$ consists of the reordered Paley spectral amplitudes $q_{k}$ according to the change of basis map specified by $i(k)=1+\sum_{j=1}^{m(k)} b_{j} 2^{n-j}$. Thus, in the Hadamard representation, the piecewise-constant structure of $f(x)$ is extracted from the vector $\mathbf{f}=\left(f_{1}, f_{2}, \ldots, f_{\mathcal{M}}\right)^{T}$, with $\tilde{\mathbf{q}}$ representing the synthesis spectrum. In this case Eq. (29) implies the forms

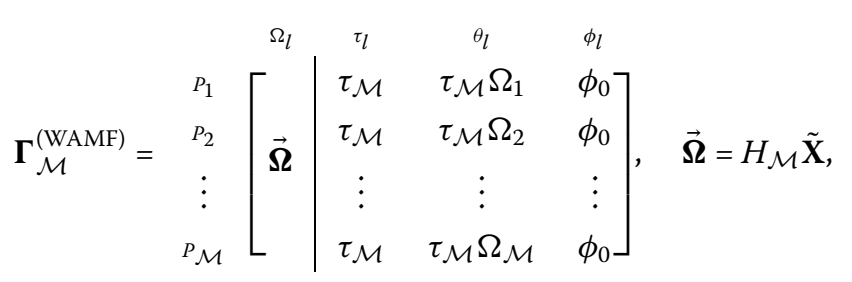




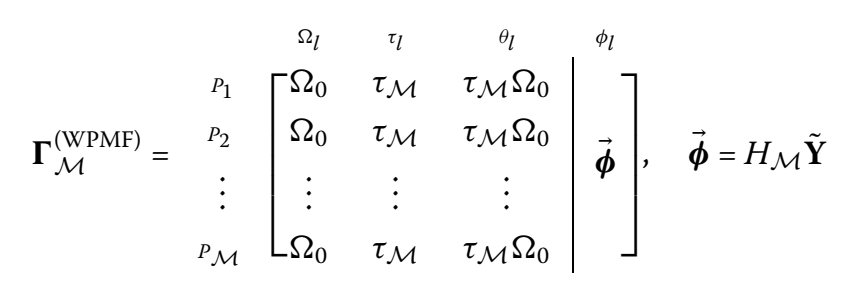

with $\mathcal{M} \equiv 2^{m(N)}$ and $\tau_{\mathcal{M}} \equiv \tau / \mathcal{M}$. The Rabi rate- or phase-modulation is thus defined by the vectors $\overrightarrow{\boldsymbol{\Omega}}=\left(\Omega_{1}, \Omega_{2}, \ldots, \Omega_{\mathcal{M}}\right)^{T}$ and $\overrightarrow{\boldsymbol{\phi}}=\left(\phi_{1}, \phi_{2}, \ldots, \phi_{\mathcal{M}}\right)^{T}$ whose components

$$
\Omega_{l} \equiv \Omega_{l}\left(X_{0}, X_{1}, \ldots, X_{N}\right), \quad \phi_{l} \equiv \phi_{l}\left(Y_{0}, Y_{1}, \ldots, Y_{N}\right), \quad l \in\{1, \ldots, \mathcal{M}\}
$$

specify the control variables during $l$ th timestep. In this case $\tau_{l}$ takes a fixed discrete value, though consecutive segments with the same values of $\Omega_{l}$ and $\phi_{l}$ may be combined sequentially to form effective operations of longer duration. The remaining degrees of freedom reside in the functional dependencies of $\Omega_{l}(\mathbf{X})$ and $\phi_{l}(\mathbf{Y})$ on the Walsh spectra, ${ }^{\mathrm{g}}$ the explicit forms of which are determined by the Hadamard matrix equations above.

The reduced control space, now compactly parameterized by the Walsh spectra, thus consists of bounded-strength unitary sequences inheriting the discrete timing properties of the Walsh basis. This contrasts with similar composite pulse methods in NMR and quantum information $[20,27,70]$ which generally rely on structures defined in continuous time. ${ }^{\mathrm{h}}$ In the next section we identify useful properties of the Walsh basis which capture filter performance and hence inform effective filter design.

\subsection{Analytic filter-design rules}

From Eqs. (31) and (32) the WAMF (WPMF) constructs are completely parameterized by the Walsh spectra $\mathbf{X}^{(i)}, i \in\{z, \Omega\}$. Here, for compactness, we denote $\mathbf{X}^{(z)}\left(\mathbf{X}^{(\Omega)}\right)=\mathbf{X}(\mathbf{Y})$. Filter properties and gate characteristics consequently map onto the basis functions in the synthesis.

To target these properties it is convenient to partition the Walsh spectrum $\mathbf{X}^{(i)} \equiv$ $\left(\mathbf{X}_{v}^{(i)}, \mathbf{X}_{\rho}^{(i)}\right)$ into spectral-amplitude classes to be treated as variational $\left(\mathbf{X}_{v}^{(i)}\right)$ and fixed parameters $\left(\mathbf{X}_{\rho}^{(i)}\right)$. Making the formal substitution $\boldsymbol{\Gamma}_{\mathcal{M}} \rightarrow \mathbf{X}^{(i)}$, the cost function in Section 4 is consequently re-expressed

$$
A_{i}\left(\mathbf{X}_{\nu}^{(i)} ; \mathbf{X}_{\rho}^{(i)}\right):=\int_{\omega_{L}}^{\omega_{c}} d \omega F_{i}\left(\omega \tau ; \mathbf{X}_{\nu}^{(i)} ; \mathbf{X}_{\rho}^{(i)}\right), \quad i \in\{z, \Omega\}
$$

where it is understood that $A_{i}$ is minimized over the space spanned by $\mathbf{X}_{v}^{(i)}$ with $\mathbf{X}_{\rho}^{(i)}$ held constant. Similarly, high-pass $(p-1)$-order filters satisfy the conditions

$$
\frac{A_{i}\left(\mathbf{X}^{(i)}\right)}{C_{2 p}\left(\mathbf{X}^{(i)}\right)}=\mathcal{O}\left(\frac{\left(\omega \tau_{c}\right)^{2 p+1}}{2 p+1}\right) \quad \Longleftrightarrow \quad C_{2}^{(i)}\left(\mathbf{X}^{(i)}\right)=\cdots=C_{2(p-1)}^{(i)}\left(\mathbf{X}^{(i)}\right)=0
$$

We are now in a position to establish a range of analytic filter-design rules to refine our search space and streamline Walsh synthesis leveraging approaches similar to electrical or digital signal filter construction. In particular, the well defined spectral properties and symmetries of the Walsh functions may be used to inform effective filter construction with improved performance. These include: 
(1) Alternate modulation quadratures for dephasing or amplitude noise.

(2) Restricting Walsh synthesis by symmetry considerations.

(3) Constraining Walsh spectra for target gate angle.

(4) Achievable filtering characteristics determined by $m(k)$ and $r(k)$.

We address each of these in turn.

(i) Alternate modulation quadratures for dephasing or amplitude noise - As the most basic element of design, we first reiterate the statements made above establishing WAMFs (WPMFs) as useful for filtering dephasing (amplitude) noise separately. In Section 9, however, we derive universal noise filters by concatenating these two filter constructs.

(ii) Restricting Walsh synthesis by symmetry considerations - As with the cosines (sines) constituting the Fourier basis, the Walsh basis separates into so-called CAL (SAL) functions with even (odd) parity. Restricting the synthesis to the CAL subset ensures the modulated waveform has time-reversal symmetry about the sequence midpoint $\tau / 2$. This can be a convenient and effective method in filter design, in line with the observation in dynamic decoupling literature $[13,71]$ that sequence performance is often improved using time-symmetric over -asymmetric building blocks. ${ }^{\mathrm{i}}$

(iii) Constraining Walsh spectra for target gate angle - Imposing desired physical properties on a candidate control sequence may generally be achieved by holding some subset $\mathbf{X}_{\rho}^{(i)}$ of the Walsh-spectral amplitudes constant. For example, we may fix the total rotation angle of the Bloch vector in order to implement a target logic operation. For WAMFs this involves a very straightforward constraint on the Walsh spectrum: the total rotation angle depends only on $X_{0}$. This can be seen as follows. First observe for Paley orders $k \geq 1$ the Walsh functions are balanced in the sense that $\int_{0}^{1} \operatorname{PAL}_{k}(x) d x=\delta_{0 k}$, where $\delta_{i j}$ denotes the Kronecker delta. For WAMFs the total gate angle $\Theta \equiv \int_{0}^{\tau} d t \Omega(t)$ therefore takes the form

$$
\Theta=\int_{0}^{\tau} \sum_{k=1}^{N} X_{k} \operatorname{PAL}_{k}(t / \tau) d t=\tau \sum_{k=1}^{N} X_{k} \int_{0}^{1} \operatorname{PAL}_{k}(x) d x=\tau \sum_{k=1}^{N} X_{k} \delta_{0 k}=X_{0} \tau .
$$

The effective gate rotation, $\theta=\Theta \bmod 2 \pi$, is consequently given by

$$
\theta=X_{0} \tau \bmod 2 \pi
$$

implying the necessary constraint on $X_{0}$ for a desired $\theta$.

(iv) Achievable filtering characteristics determined by $m(k)$ and $r(k)$ - The achievable filter order over the entire stopband is essentially limited by the number of constituent control operations: one may achieve higher $p$ at the cost of higher $n$. For the Walshsynthesized filters in Eqs. (31) and (32), with $N$ the highest-order basis function, $n \equiv 2^{m(N)}$. Hence higher-order Walsh functions generally produce higher-order filters.

For high-pass filters further insight is gained by examining the low-frequency spectral properties of the $\operatorname{PAL}_{k}(t / \tau)$. This reflects the fact that the filter-transfer functions are closely related to Fourier transforms of relevant time-domain control functions. In particular, the Fourier transform of $\operatorname{PAL}_{k}(t / \tau)$, near zero frequency, has a power-law expansion [51]

$$
\mathcal{F}_{x}\left[\operatorname{PAL}_{k}(x)\right] \propto(\omega \tau)^{r(k)},
$$


where $r(k)$ is the Hamming weight. Here $x \equiv t / \tau$ is a non-dimensional time-domain variable and $\mathcal{F}_{x}\left[\operatorname{PAL}_{k}(x)\right]$ denotes the forward Fourier transform of $\operatorname{PAL}_{k}(x)$ from $x$ to the (non-dimensional) angular frequency variable $\omega \tau$ in Fourier space. Increasing the lowfrequency roll-off is therefore associated with maximizing $r(k)$ for a given number of control operations $n=2^{m(k)}$. This corresponds to maximizing the number of Rademacher functions in the construction ${ }^{j}$ and immediately identifies Paley orders $k=2^{\alpha}-1, \alpha \in \mathbb{N}$, (see Figure 2) as key design resources.

\section{Walsh Amplitude Modulated Filters (WAMFs)}

Having introduced the basic physical picture and mathematical basis for Walsh filter synthesis, we move on to demonstrate explicit realizations of WAMFs for dephasing noise. Both first and second-order filters with high-pass filter characteristics are constructed.

\subsection{First-order WAMFs}

We begin by considering first-order filters for dephasing noise implementing target singlequbit rotations. Construction begins by considering the design rules (i)-(iv) outlined in Section 5.3. For filtering noise in this quadrature (i) implies we should employ the WAMF construction (Eq. (31)). In this case, invoking (iii), the requirement of implementing nontrivial gates dictates we include Paley order $k=0$ in the synthesis. The average Rabi rate (and hence rotation angle) is then determined by $X_{0}$, the spectral amplitude of $\operatorname{PAL}_{0}(t / \tau)$. The remaining synthesis choices include basis functions of Paley order $k>0$ and are in principle unbounded.

As a first application, we pursue the construction minimizing the number $n=2^{m(N)}$ of unitary operations in the synthesized sequence such that error suppression is still attainable. In line with design rules (ii) and (iv), time-reversal symmetry is ensured and the number of Rademacher functions is maximized by reducing the remaining synthesis choices to the single basis function $\operatorname{PAL}_{3}(t / \tau)$ (Figure 2). Hence, in this simple example, $N=3$, and $\mathcal{M}(N)=2^{m(N)}=2^{2}$, yielding 4-segment gates with segment lengths $\tau_{\mathcal{M}}=\tau / 4$. These represent the lowest-order constructions with error suppression capabilites.

The Rabi rate is consequently written $\Omega(t)=X_{0} \operatorname{PAL}_{0}(t / \tau)+X_{3} \operatorname{PAL}_{3}(t / \tau)$. Physically, $X_{3}$ specifies the modulation depth of the resulting Rabi rate envelope (see inset to Figure 3(c)) while $X_{0}$ determines the average value as described above. Accordingly, for a particular target rotation, we treat $X_{0}$ as a fixed parameter (see Eq. (36)) while $X_{3}$ is treated as a variational parameter by which to optimize the (dephasing) cost function (Eq. (34)). Thus, values of $X_{3}$ for which $A_{z}\left(X_{3} ; X_{0}\right)$ is minimized specify the optimum modulation depths for an effective filter.

Using Eq. (30) the Walsh synthesis spectrum is represented $\tilde{\mathbf{X}} \equiv\left(\tilde{X}_{1}, \tilde{X}_{2}, \tilde{X}_{3}, \tilde{X}_{4}\right)^{T}=$ $\left(X_{0}, 0,0, X_{3}\right)^{T}$, yielding the Hadamard representation of the modulated Rabi rate

$$
\overrightarrow{\boldsymbol{\Omega}}=\left[\begin{array}{cccc}
1 & 1 & 1 & 1 \\
1 & -1 & 1 & -1 \\
1 & 1 & -1 & -1 \\
1 & -1 & -1 & 1
\end{array}\right]\left[\begin{array}{c}
X_{0} \\
0 \\
0 \\
X_{3}
\end{array}\right]=\left[\begin{array}{l}
X_{0}+X_{3} \\
X_{0}-X_{3} \\
X_{0}-X_{3} \\
X_{0}+X_{3}
\end{array}\right]
$$




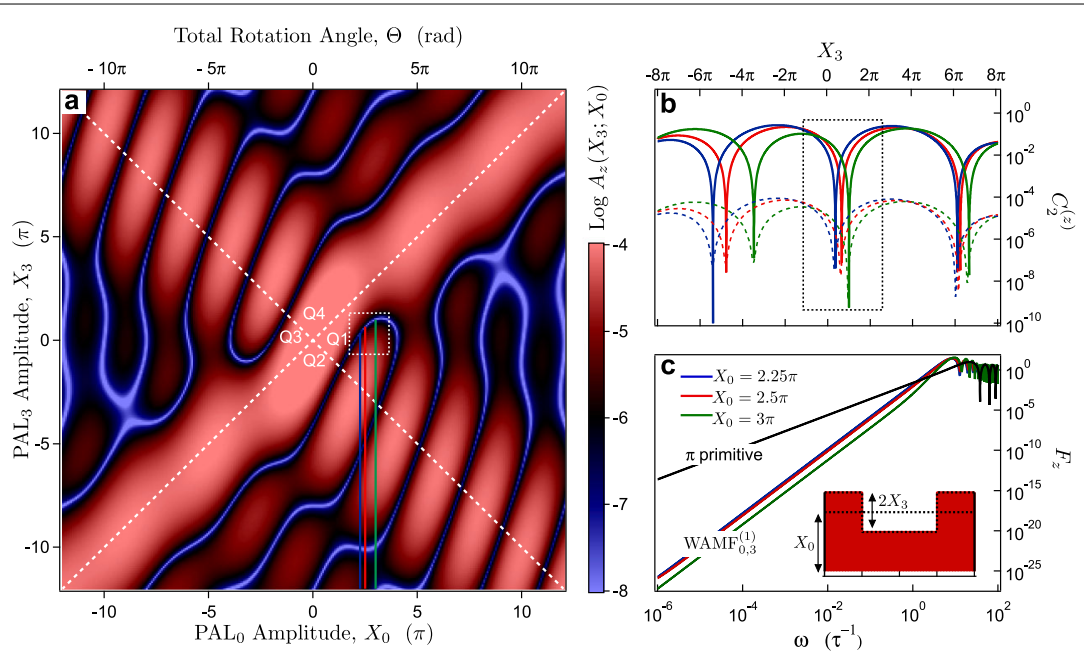

Figure 3 First-order WAMFs. Construction of WAMF $\mathrm{WA}_{0,3}^{(1)}$ for dephasing noise filtering. (a) Log-scale color plot of the cost function $A_{z}\left(X_{3} ; X_{0}\right)$ integrated over $\omega \in\left[10^{-9}, 10^{-1}\right] \tau^{-1}$. Total gate angle $\Theta=X_{0}(\tau \equiv 1)$. Blue regions indicate minima in $A_{z}\left(X_{3} ; X_{0}\right)$, implying optimized filter synthesis. Coloured lines (blue, red, green) at $X_{0}=\left(2 \frac{1}{4}, 2 \frac{1}{2}, 3\right) \pi$ correspond to rotation angles $\theta=\left(\frac{\pi}{4}, \frac{\pi}{2}, \pi\right)$. These lines terminate at values

$X_{3}=(0.36 \ldots, 0.65 \ldots, 1) \pi$ on a blue contour (boxed) and indicate representative points in the $X_{0} X_{3}$ plane for which first-order filtering is achieved. In this plot, for $\left|X_{3}\right|>\left|X_{0}\right|$ the Rabi rates $X_{ \pm}$have opposite sign, implying a $\pi$-phase shift in addition to amplitude modulation (e.g. see Eq. (145)). We therefore distinguish quadrants Q1 and Q3 in the $X_{0} X_{3}$ plane in which $\left|X_{3}\right| \leq\left|X_{0}\right|$ (strict amplitude modulation) and Q2 and Q4 in which $\left|X_{3}\right|>\left|X_{0}\right|$ (sign-switching amplitude modulation). (b) Solid lines: first order Taylor coefficient $C_{2}^{(z)}\left(X_{3} ; X_{0}\right)$ as a function of $X_{3}$ with $X_{0}=\left(2 \frac{1}{4}, 2 \frac{1}{2}, 3\right) \pi$; zeros appear as dips on log-scale. Dotted lines: one-dimensional slices of $A_{z}\left(X_{3} ; X_{0}\right)$ for same fixed values of $X_{0}$. Boxed dips correspond to boxed points in a) where the colored lines intersect with the blue contour. (c) Filter-transfer functions for the spectrally optimized $\mathrm{WAMF}_{0,3}^{(1)}$ gates identified by the boxed features in (a) and (b).

The resulting WAMF construction, denoted $\mathrm{WAMF}_{0,3}^{(1)}$, is thus represented

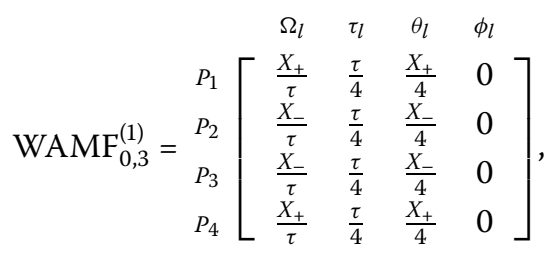

where $X_{ \pm}:=X_{0} \pm X_{3}$, and the superscript in this notation denotes first-order filter capabilities. Hence these gates inhabit the two-dimensional control space spanned by the $X_{0} X_{3}$ plane (see Figure 3(a)). The dephasing filter-transfer function $F_{z}\left(\omega ; X_{3} ; X_{0}\right)$ for an arbitrary WAMF $_{0,3}^{(1)}$ gate is derived by substituting Eq. (39) into Eq. (13). The cost function $A_{z}\left(X_{3} ; X_{0}\right)$ may then be numerically integrated.

Figure 3(a) shows a two-dimensional representation of $A_{z}\left(X_{3} ; X_{0}\right)$ integrated over the stopband $\omega \in\left[10^{-9}, 10^{-1}\right] \tau^{-1}$. The value of $\log _{10}\left[A_{z}\left(X_{3} ; X_{0}\right)\right]$ is indicated by the color scale. Total sequence length is normalized to $\tau=1$ in this data, so the total gate rotation angle $\Theta \equiv X_{0}$ is given directly by the $X_{0}$-axis. As can be seen, for any fixed $X_{0}$ there exist quasi-periodic tunings of $X_{3}$ which minimize the cost function. In other words, we have a prescription for synthesizing spectrally-optimized dephasing filters which implement arbitrary rotation angles. Interestingly, the point $\left(X_{0}, X_{3}\right) \equiv(3 \pi, \pi)$ reproduces the previously derived first-order DCG NOT construction [19]. 
Blue regions, where the cost function has been minimized, represent first-order filters for low-frequency noise due to the restrictions placed on the synthesis space. ${ }^{\mathrm{k}}$ To demonstrate that these optimized WAMF $\mathrm{W}_{0,3}^{(1)}$ gates perform as first-order filters we Taylor expand $F_{z}\left(\omega ; X_{3} ; X_{0}\right)$ as in Eq. (35), and derive an easy analytic expression for the first order coefficient

$$
C_{2}^{(z)}\left(X_{3} ; X_{0}\right)=4\left[\frac{\left(X_{0}-X_{3}\right) \sin \left(\frac{X_{0}}{2}\right)+2 X_{3} \sin \left(\frac{X_{0}-X_{3}}{4}\right)}{\left(X_{0}^{2}-X_{3}^{2}\right)}\right]^{2} .
$$

In principle we may now solve $C_{2}^{(z)}\left(X_{0} ; X_{3}\right)=0$ to find values of $X_{3}$ giving first-order filters for a given $X_{0}$. In Figure 3 (b) we plot $C_{2}^{(z)}\left(X_{0} ; X_{3}\right)$ (solid lines) as a function of $X_{3}$ for the choices $X_{0}=\left(2 \frac{1}{4}, 2 \frac{1}{2}, 3\right) \pi$ as above. Zeros of $C_{2}^{(z)}\left(X_{0}, X_{3}\right)$, appearing as dips on the log scale, occur quasi-periodically in $X_{3}$ and match with points in Figure 3(a) where corresponding lines of constant $X_{0}$ intersect with the blue contours. To demonstrate this we plot one-dimensional slices of $A_{z}\left(X_{3} ; X_{0}\right)$ for fixed values $X_{0}=\left(2 \frac{1}{4}, 2 \frac{1}{2}, 3\right) \pi$ (dotted lines). We find the minima in $A_{z}\left(X_{3} ; X_{0}\right)$ align with zeros of $C_{2}^{(z)}\left(X_{0} ; X_{3}\right)$, implying the blue contours in the $X_{0} X_{3}$ plane do indeed produce first-order filters, with $(p-1)=1$. The boxed zeros near $X_{3}=(0.36 \ldots, 0.65 \ldots, 1) \pi$ correspond to the termination points of the colored lines in Figure 3(a) (also boxed). These indicate representative points in the $X_{0} X_{3}$ plane producing first-order filters with nontrivial rotations. In particular, these filters implement $\theta=\left(\frac{\pi}{4}, \frac{\pi}{2}, \pi\right)$ rotations.

The corresponding dephasing filter-transfer functions for these three optimized gates are shown in Figure 3(c). As expected from Eq. (21), with $C_{2}^{(z)}=0$, these approximately satisfy $F_{z} \propto(\omega \tau)^{4}$ in the stopband, producing first-order filters with $(p-1)=1$. For comparison we include the dephasing filter-transfer function for a primitive $\pi$ rotation where $F_{z} \propto(\omega \tau)^{2}$, implying $(p-1)=0$. The steeper slopes, or roll-offs, for the optimized $\mathrm{WAMF}_{0,3}^{(1)}$ gates captures this difference. This filter design method, and the performance of the $\mathrm{WAMF}_{0,3}^{(1)}$ filters, has recently been experimentally validated by our group [37].

\subsection{Second-order WAMFs}

We now consider higher-order dephasing filters by increasing the number $n$ of segments in the sequence. In particular we consider 8-segment gates. Construction again begins by considering the design rules (i)-(iv) outlined in Section 5.3.

Using (i) and (iii) we employ the WAMF construction and include Paley order $k=0$ to ensure nontrivial rotation angles. Extending to 8-segments, however, increases the accessible range of Walsh functions in the synthesis as identified in design rule (iv). Specifically we extend the synthesis to Paley orders $k \leq 7$ corresponding to the complexity class $m(k) \leq 3$, implying a $2^{3}=8$ segments construction in the Hadamard representation. We denote these constructions by $\mathrm{WAMF}_{0: 7}^{(2)}$ where the superscript indicates second-order filtering capabilities, as will be shown. Imposing time-reversal symmetry about $\tau / 2$ further restricts the synthesis space to $k \in\{3,5,6\}$, corresponding to CAL functions referenced in design rule (ii). We therefore study synthesized filters with spectral amplitudes partitioned into fixed $\mathbf{X}_{\rho}=X_{0}$ and variational $\mathbf{X}_{v}=\left(X_{3}, X_{5}, X_{6}\right)$ classes.

As a representative example we set $X_{0}=3 \pi$ and restrict attention to filters implementing a net $\pi$ rotation $(\tau \equiv 1)$. Our cost function consequently takes the form $A_{z}\left(X_{3}, X_{5}, X_{6} ; 3 \pi\right)=\int_{\omega_{L}}^{\omega_{c}} F_{z}\left(\omega \tau ; X_{3}, X_{5}, X_{6} ; 3 \pi\right) d \omega$, implying a three-dimensional varia- 


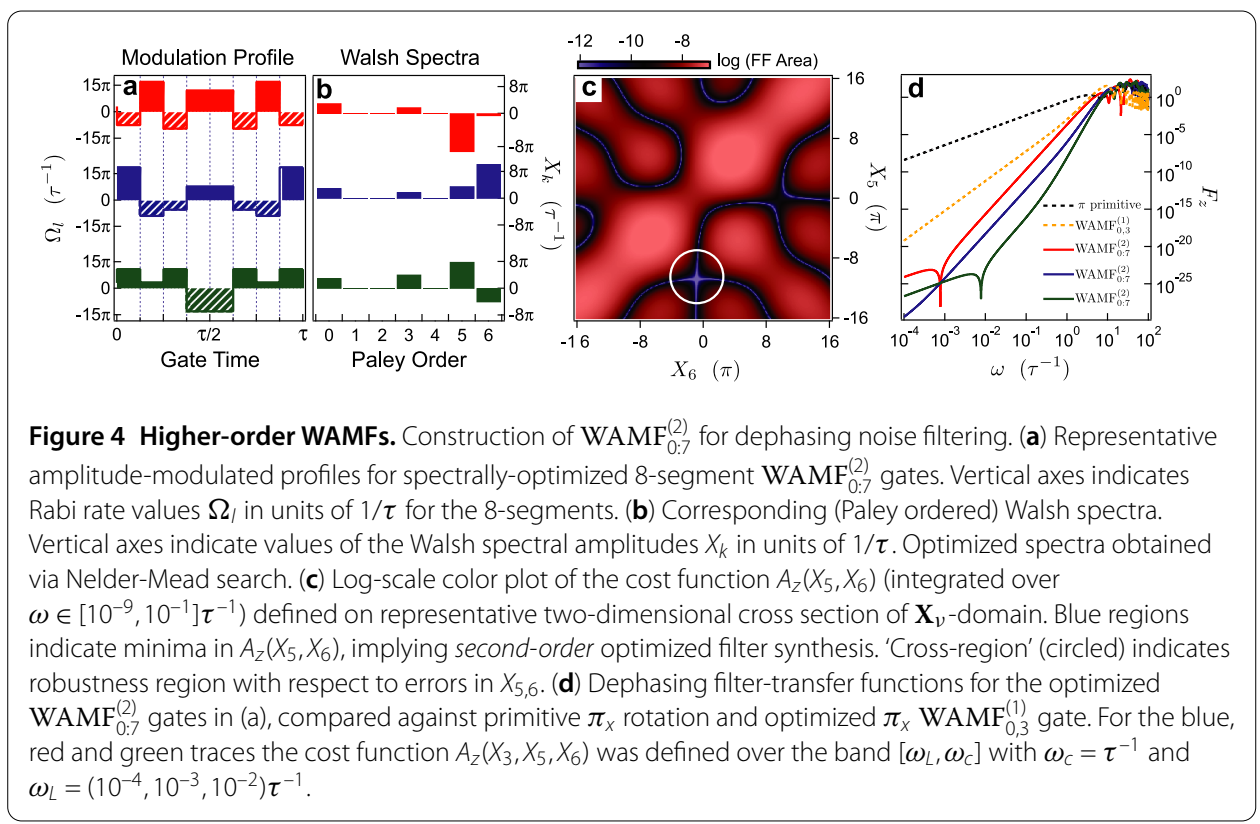

tional control space over which to derive spectrally-optimized filters. We accomplish this using a Nelder-Mead search to minimize $A_{z}\left(\mathbf{X}_{v} ; 3 \pi\right)$ over the $\mathbf{X}_{v}$-domain.

Representative examples of spectrally-optimized $\mathrm{WAMF}_{0: 7}^{(2)}$ constructions are shown in Figure 4. The 8-segment time-domain amplitude-modulated profiles are represented in Figure 4(a), with corresponding Walsh spectra shown in Figure 4(b). The blue, red and green spectra were obtained using a Nelder-Mead optimization of the cost function $A_{z}\left(X_{3}, X_{5}, X_{6}\right)$ defined over $\left[\omega_{L}, \omega_{c}\right]$ with $\omega_{c}=\tau^{-1}$ and $\omega_{L}=\left(10^{-4}, 10^{-3}, 10^{-2}\right) \tau^{-1}$ respectively. The corresponding dephasing filter-transfer functions $F_{z}(\omega)$ are plotted as solid blue, red and green traces in Figure 4(d). Within the respective cost function bands these satisfy $F_{z} \propto(\omega \tau)^{2 p^{*}}$, with the instantaneous filter order ranging between $2<\left(p^{*}-1\right)<3.8$ at various points. ${ }^{1}$ For comparison we also plot the dephasing filter-transfer function for a primitive $\pi$ rotation (black dashed trace) and an optimized $\mathrm{WAMF}_{0,3}^{(1)}$ gate (yellow dashed trace). These respectively satisfy $F_{z} \propto(\omega \tau)^{2,4}$ over the whole stopband and are well characterized by the (asymptotic) filter orders $(p-1)=0,1$.

Figure 4(a) shows a two-dimensional representation of $A_{z}\left(X_{5}, X_{6}\right)$ defined on a twodimensional cross-section of the $\mathbf{X}_{v}$-domain, holding $X_{3}$ fixed, and integrated over the stopband $\omega \in\left[10^{-9}, 10^{-1}\right] \tau^{-1}$. The value of $\log _{10}\left[A_{z}\left(X_{5}, X_{6}\right)\right]$ is indicated by the color scale. Areas in blue indicate minima in $A_{z}\left(X_{5}, X_{6}\right)$, indicating optimized paths in $X_{5} X_{6}$ plane the over which effective filters may be found. Notably, it is possible to find 'cross-regions' (circled) in which the spectral amplitudes $X_{5}$ and $X_{6}$ may independently be varied substantially without the cost function moving off a local minima. This potentially indicates the existence of classes of WAMFs which may be robust to errors in the Walsh spectrum itself.

\section{Walsh Phase Modulated Filters (WPMFs)}

We now turn to filters for amplitude-damping noise constructed via phase modulation using the WPMF construction set out in Eq. (32). Following the same procedure described above for WAMFs, one can implement a Nelder-Mead search to derive spectrallyoptimized WPMFs which implement nontrivial rotations. For these constructions, however, the target rotation angle is dependent on both the Rabi rate and the sequence of 
phase modulations. Consequently it is less straightforward to impose a constraint during the optimization procedure to ensure a particular target rotation. Although we do not pursue the general problem in detail in this paper, we demonstrate the approach in this and the following sections, deriving a family of WPMFs in which the synthesis space is limited to a variety of simple combinations of Walsh function.

In the remainder of this section we study a variation on the strict WPMF structure which resolves the difficulty of imposing a target rotation and enables us to make some useful connections with existing composite pulse sequences in NMR. This variation involves partitioning the control modulation into a target rotation $P(\theta, 0)$ followed by a sequence of phase-modulated identity operations $\prod_{l=1}^{\mathcal{M}} P\left(2 \pi, \phi_{l}\right)$ with the $\phi_{l}$ chosen such that amplitude noise is filtered to some order. Here the operator $P(\theta, \phi)$ denotes the rotation through angle $\theta$ about $\hat{\sigma}_{\phi}$. By insisting these $\mathcal{M}$ 'correction' segments are all identity operations, the phase modulations do not produce complicated rotation paths and the net rotation is determined simply by the initial target pulse.

We assume a constant Rabi rate $\Omega_{0}$ so that each correction segment has equal duration $\tau_{2 \pi}=2 \pi / \Omega_{0}$. Provided $\mathcal{M}$ is a power of 2 , the phase modulation describing the correction sequence may therefore be constructed as a Walsh-synthesized waveform consisting of Paley orders $k \leq \mathcal{M}-1$. The simplest such 'synthesis' derives from a single Walsh function $\operatorname{PAL}_{k}(x)$ with spectral weight $Y_{k}$, yielding the sequence

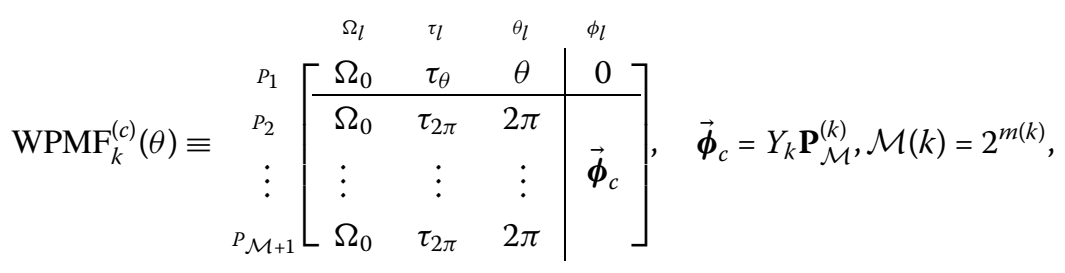

where, as in Eq. (25), the column vector $\mathbf{P}_{\mathcal{M}}^{(k)}$ specifies the sequence of values taken by $\operatorname{PAL}_{k}\left(\frac{t-\tau_{\theta}}{\tau-\tau_{\theta}}\right)$ over the interval $\left[\tau_{\theta}, \tau\right]$ partitioned into the minimum $\mathcal{M}(k)$ equal time bins. We include the superscript $(c)$ and write the vectorized phase $\overrightarrow{\boldsymbol{\phi}}_{c}$ to indicate Walsh modulation during the 'correction stage' of the sequence, disambiguating this from the strict WPMF structure. For a given $\theta$ we may now treat $Y_{k}$ as a tuning parameter which may be optimized by minimizing the cost function $A_{\Omega}\left(Y_{k} ; \theta\right) \equiv \int_{0}^{\omega_{c}} d \omega F_{\Omega}\left(\omega \tau ; Y_{k} ; \theta\right)$. The optimized $Y_{k}$ are thereby defined as an implicit function of $\theta$.

In fact we may analytically show this construction yields first-order filters for amplitude noise by Taylor expanding $F_{\Omega}(\omega)$ and solving the first-order filter condition $C_{2}^{(\Omega)}\left(Y_{k} ; \theta\right)=0$. We compute $C_{2}^{(\Omega)}\left(Y_{k} ; \Omega\right)=\left[\theta+2 \pi \mathcal{M}(k) \cos \left(Y_{k}\right)\right]^{2} / 4$, implying the optimized Walsh spectral amplitude

$$
Y_{k}(\theta)=\cos ^{-1}\left(-\frac{\theta}{2 \pi \mathcal{M}(k)}\right) .
$$

On the other hand, computing the second-order Taylor coefficient and substituting in the first-order-optimized spectral value in Eq. (42), one finds $C_{4}^{(\Omega)} \neq 0$. Hence the $\mathrm{WPMF}_{k}^{(c)}$ sequence has $(p-1)=1$ filtering properties.

We make the interesting observation that Eqs. (41) and (42) produce the first-order Solovay-Kitaev SK1 $(\theta)$ sequence $[72,73]$ and the second-order Wimperis passband P2 $(\theta)$ 
Table 1 Filter characteristics of $\mathrm{WPMF}_{k}^{(c)}$ constructions corresponding to well-known NMR sequences, SK1 and P2, originally designed to compensate for static amplitude errors to first and second Magnus order respectively (Section 4.3)

\begin{tabular}{llllllll}
\hline & \multicolumn{3}{l}{ WPMF $_{k}^{(c)}$ construction } & & \multicolumn{2}{l}{ Amplitude errors } \\
\cline { 2 - 4 } & $k$ & $\mathcal{M}(k)$ & $\overrightarrow{\boldsymbol{\phi}}$ & & & $(\mu-1)$ & $(p-1)$ \\
\hline SK1 $(\theta)$ & 1 & 2 & $Y_{1}(\theta) \mathrm{PAL}_{1}$ & $\cos ^{-1}(-\theta / 4 \pi)$ & & 1 \\
$\operatorname{P2}(\theta)$ & 3 & 4 & $Y_{3}(\theta) \mathrm{PAL}_{3}$ & $\cos ^{-1}(-\theta / 8 \pi)$ & & 2 & 1 \\
\hline
\end{tabular}

sequence [27] by setting $k=1,3$ respectively. Hence these well-known NMR sequences, originally designed to compensate for static amplitude errors to first and second Magnus order respectively (see Section 4.3), appear in the Walsh filter space as phase-modulated filters for non-Markovian amplitude noise. Table 1 summarizes this.

Another remarkable result is found using the synthesis $\overrightarrow{\boldsymbol{\phi}}_{c}=H_{\mathcal{M}} \tilde{\mathbf{Y}}$ over the two Walsh functions $\mathrm{PAL}_{0}$ and $\mathrm{PAL}_{3}$, setting $\tilde{\mathbf{Y}} \equiv\left(Y_{0}, 0,0, Y_{3}\right)^{T}$ in analogy with the Walsh spectrum defining amplitude modulation in the $\mathrm{WAMF}_{0,3}^{(1)}$ construction. The first-order filtering condition $C_{2}^{(\Omega)}\left(Y_{0}, Y_{3}, \Omega_{0} ; \theta\right)=0$ then implies solutions

$$
\tilde{\mathbf{Y}}=\left(2 \phi_{\mathrm{BB} 1}, 0,0,-\phi_{\mathrm{BB} 1}\right)^{T}, \quad \phi_{\mathrm{BB} 1}(\theta) \equiv \cos ^{-1}\left(-\frac{\theta}{4 \pi}\right), \quad \Omega_{0}=\frac{4 \pi+\theta}{\tau}
$$

yielding the Wimperis broadband BB1 sequence [74]

$$
\operatorname{BB} 1(\theta)=\begin{gathered}
P_{1} \\
P_{2} \\
P_{3} \\
P_{4} \\
P_{5}
\end{gathered}\left[\begin{array}{cccc}
\Omega_{l} & \tau_{l} & \theta_{l} & \phi_{l} \\
\Omega_{0} & \tau_{\theta} & \theta & 0 \\
\Omega_{0} & \frac{\tau-\tau_{\theta}}{4} & \pi & Y_{+} \\
\Omega_{0} & \frac{\tau-\tau_{\theta}}{4} & \pi & Y_{-} \\
\Omega_{0} & \frac{\tau-\tau_{\theta}}{4} & \pi & Y_{-} \\
\Omega_{0} & \frac{\tau-\tau_{\theta}}{4} & \pi & Y_{+}
\end{array}\right] \cong \begin{gathered}
P_{1} \\
P_{2} \\
P_{3} \\
P_{4}
\end{gathered}\left[\begin{array}{cccc}
\Omega_{0} & \tau_{\theta} & \theta & \theta_{l} \\
\Omega_{0} & \frac{\tau-\tau_{\theta}}{4} & \pi & \phi_{\mathrm{BB} 1} \\
\Omega_{0} & \frac{\tau-\tau_{\theta}}{2} & 2 \pi & 3 \phi_{\mathrm{BB} 1} \\
\Omega_{0} & \frac{\tau-\tau_{\theta}}{4} & \pi & \phi_{\mathrm{BB} 1}
\end{array}\right] .
$$

Here $Y_{ \pm}:=Y_{0} \pm Y_{3}$ and in the last equality we have collapsed the array to show the BB1 construction explicitly. Computing the second-order Taylor coefficient and in substituting the first-order-optimized spectral values, however, we find $C_{4}^{(\Omega)} \neq 0$. Thus, although BB1 was originally derived to compensate static amplitude errors to second Magnus order, it only provides first-order noise filtering.

\section{Walsh Rotary Spin Echo (WRSE)}

In this section we treat a sub-class of Walsh modulated filters which may be described either in terms of phase- or amplitude-modulation. The phase-modulation consists of applying a sequence of $\pi$-phase shifts, relative to some offset $\phi_{0}$, on the driving field with a constant amplitude $\Omega_{0}$. This construction generalizes the rotary spin echo (RSE) sequence from NMR, analogous to the Hahn-echo sequence for driven systems, consisting of a single $\pi$-phase shift applied at the sequence midpoint $\tau / 2$. In quantum information RSE has been employed, for example, in relaxation noise spectroscopy [75] and in mitigating lowfrequency off-resonant noise [76] in driven superconducting flux qubits. In contrast with previous approaches, our generalization permits higher-order filter performance in both amplitude and dephasing quadratures and may prove of significant use.

In our construction the temporal profile of the phase is expressed $\phi(t)=\phi_{0}+\frac{\pi}{2}(1-$ $y(t))$, where $y(t) \in\{ \pm 1\}$ is a binary-valued switching function defined to change sign at the 

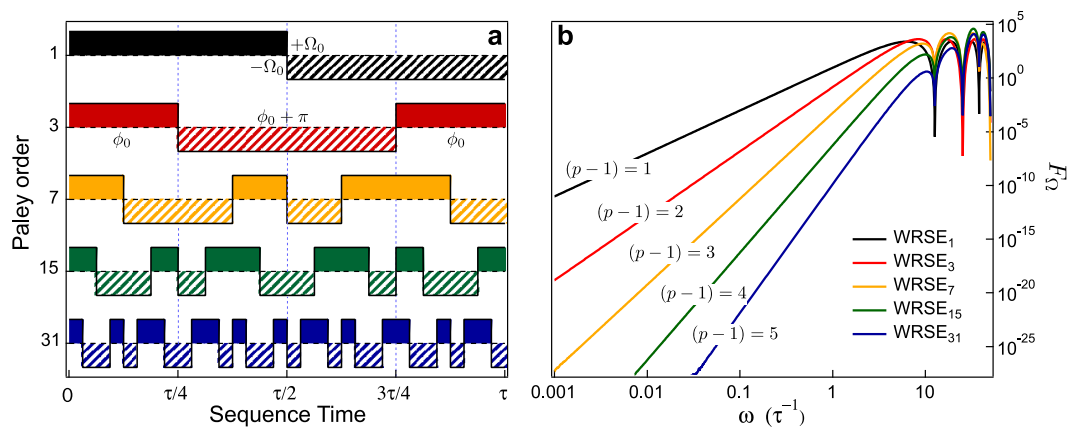

Figure 5 WRSEs as amplitude-noise filters. WRSE $E_{k}$ amplitude noise filter characteristics, $k \in\{1,3,7,15,31\}$. (a) Modulation profiles of $\mathrm{WRSE}_{k}$ sequences. Amplitude modulation involves switching the sign $\pm \Omega_{0}$; this is equivalent to holding $\Omega_{0}$ constant and instead shifting the phase $\phi_{0}$ by $\pi$, indicated by the hatching. (b) Corresponding amplitude filter-transfer functions $F_{\Omega}(\omega)$, showing filter order increases with Hamming weights $r(k) \in\{1,2,3,4,5\}$.

application of each $\pi$-shift. Specifically, we consider sequences based on Walsh functions by defining the switching function

$$
y(t):=\operatorname{PAL}_{k}(t / \tau), \quad t \in\{0, \tau\} .
$$

Consequently the phase has Walsh synthesis $\phi(t)=Y_{0} \operatorname{PAL}_{0}(t / \tau)+Y_{k} \operatorname{PAL}_{k}(t / \tau)$ where $Y_{0}=\phi_{0}+\frac{\pi}{2}$ and $Y_{k}=-\frac{\pi}{2}$. These gates only perform the identity operation with $\Theta \equiv 0$ owing to the property that $\hat{\sigma}_{\phi_{0}+\pi}=-\hat{\sigma}_{\phi_{0}}$ (see Eq. (6)). This implies the direction of unitary rotation is reversed by the application of each $\pi$ shift, and since any Walsh function of Paley order $k>0$ is equally distributed between values \pm 1 over the domain these rotations perfectly cancel, yielding zero total rotation. This is formally equivalent to modulating the sign of the Rabi rate and holding the phase $\phi_{0}$ constant (see Appendix C), as schematically illustrated in Figure 5(a). These sequences, referred to as Walsh rotary spin echo order $k$ (WRSE ${ }_{k}$ ), thus take the form

$$
\mathrm{WRSE}_{k} \equiv \prod_{l=1}^{\mathcal{M}(k)} \exp \left(i \frac{\Omega_{0}}{2} \tau_{\mathcal{M}} \hat{\sigma}_{\phi_{l}}\right), \quad \overrightarrow{\boldsymbol{\phi}}=\left(\phi_{1}, \phi_{2}, \ldots, \phi_{\mathcal{M}}\right)^{T}=H_{\mathcal{M}} \tilde{\mathbf{Y}}
$$

where, referring to Eqs. (32) and (30), $\mathcal{M}(k) \equiv 2^{m(k)}$ and the Walsh spectrum $\tilde{\mathbf{Y}}$ has only two nonzero elements: $\tilde{Y}_{1}=\phi_{0}+\frac{\pi}{2}$ and $\tilde{Y}_{i(k)}=-\frac{\pi}{2}$. This naming convention reflects the fact that the WRSE family generalize the traditional RSE sequence: in particular, WRSE ${ }_{1} \equiv$ RSE. We now proceed to derive the filtering properties of the WRSE family, starting with the amplitude quadrature then moving to the dephasing quadrature.

\subsection{WRSE as amplitude filters}

The amplitude filter-transfer function, in the stopband, is determined by the Hamming weight of the chosen Walsh function by

$$
F_{\Omega}(\omega \tau) \propto(\omega \tau)^{2(r(k)+1)} .
$$

Comparing this with the low-frequency approximation $F_{\Omega}(\omega \tau ; k) \propto(\omega \tau)^{2 p}$ from Eq. (22), we conclude the time-domain filter order is given by $(p-1)=r(k)$. That is, high-pass filter 
performance is completely determined by the Hamming weight. Figure 5(c) demonstrates this by plotting $F_{\Omega}(\omega)$ for the $\mathrm{WRSE}_{k}$ sequences with $k \in\{1,3,7,15,31\}$, corresponding to the Hamming weights $r(k) \in\{1,2,3,4,5\}$. The corresponding filter order increase is clear from the steepening roll-off.

This result follows from deriving $F_{\Omega}(\omega)$ for Eq. (46) which, owing to the fact that the noise Hamiltonian in this quadrature always commutes with control operations, takes the relatively simple analytical form (see Appendix C)

$$
F_{\Omega}(\omega)=\frac{\Omega_{0}^{2}}{4}\left|\sum_{l=1}^{\mathcal{M}} P_{l}^{(k)}\left[e^{i \omega t_{l-1}}-e^{i \omega t_{l}}\right]\right|^{2} .
$$

Our key insight now is to observe the sum inside the modulus square above satisfies $\sum_{l=1}^{\mathcal{M}} P_{l}^{(k)}\left[e^{i \omega t_{l}}-e^{i \omega t_{l-1}}\right]=i \omega \tau \mathcal{F}_{x}\left[\operatorname{PAL}_{k}(x)\right]$, where $x \equiv t / \tau$ is a non-dimensional timedomain variable. We now invoking Eq. (37) in design rule (iii), namely $\mathcal{F}_{x}\left[\operatorname{PAL}_{k}(x)\right] \propto$ $(\omega \tau)^{r(k)}$, to map the low-frequency spectral properties onto the Hamming weight $r(k)$ of the chosen Walsh function. Substituting this into Eq. (48) then yields Eq. (47).

\subsection{WRSE as dephasing filters}

The dephasing filter performance for $\mathrm{WRSE}_{k}$ is more complicated to study as noise terms in this quadrature do not commute with our control, obfuscating a compact expression for $F_{z}(\omega)$. It is convenient instead to study the zeros of the Taylor coefficients $C_{2 j}^{(z)}$ as in Eq. (22). Since the Rabi rate is the only free variable in the $\mathrm{WRSE}_{k}$, for a given $k$, it follows the Taylor coefficients are functions only of $\Omega_{0}$. Filtering to order $(p-1)$ then corresponds to the condition

$$
C_{2}^{(z)}(\eta ; k)=C_{4}^{(k)}(\eta ; k)=\cdots=C_{2(p-1)}^{(z)}(\eta ; k)=0,
$$

where $\eta$ denotes some value of $\Omega_{0}$ for which the above coefficients are concurrently zero. Here we have included the Paley order as a parameter of the coefficients. Analysis shows, however, concurrent zeros exist only for $j \in\{1,2\}$. We effectively obtain the following 'nogo theorem': $\mathrm{WRSE}_{k}$ sequences perform as (high-pass) dephasing noise filters up to (but not beyond) second order. This result may be of use to characterize the relevant quadrature of an unknown noise source, by probing with higher-order $\mathrm{WRSE}_{k}$ sequences and determining the resulting filtering properties.

The general insight supporting this statement is developed in Appendix $\mathrm{C}$, by explicitly studying the representative case for $\mathrm{WRSE}_{3}$. The Taylor coefficients $C_{2,4,6}^{(z)}$ for this representative case are plotted in Figure 6(a) as functions of $\Omega_{0}$. Zeros of $C_{2}^{(z)}$ occur at multiples of $4 \pi$, and concurrent zeros of $C_{2,4}^{(z)}$ occur at multiples of $8 \pi$. However, since $C_{6}^{(z)}$ is never zero at multiples of $8 \pi$ (see inset to Figure 6(a)), it follows we can never achieve higher than second-order filtering. We verify this by examining the slope of $F_{z}\left(\omega ; \Omega_{0}\right)$ for the values $\Omega_{0}=2 \pi q, q \in\{1, \ldots, 8\}$ (see Figure 6(b)). Similar considerations for other values of $k$ generalize the result.

\section{Universal Walsh Modulated Filters (UWMFs)}

In the previous sections we have considered WAMF and WPMF gates which implement target qubit rotations while filtering, to some order, either dephasing or amplitude noise 

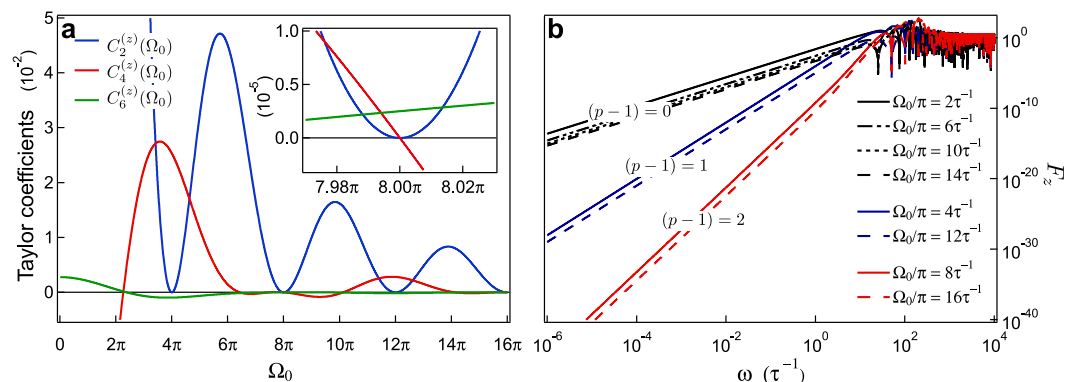

Figure 6 WRSEs as dephasing-noise filters. WRSE 3 dephasing noise filter characteristics. (a) Taylor expansion coefficients $C_{2,4,6}^{(z)}\left(\Omega_{0} ; 3\right)$ for $F_{z}(\omega)$. The inset shows typical behaviour: $\Omega_{0}=8 \pi$ is a concurrent zero of $C_{2,4}^{(z)}\left(\Omega_{0} ; 3\right)$, but not of $C_{6}^{(z)}\left(\Omega_{0} ; 3\right)$. Hence WRSE 3 can only filter up to second-order. (b) Dephasing filter-transfer functions for WRSE $\mathrm{WR}_{3}$ corresponding to $\Omega_{0}=2 \pi q, q \in\{1, \ldots, 8\}$. When $\Omega_{0}$ is a multiple of $8 \pi$ we achieve second-order filters, that is $(p-1)=2$.

respectively. In this section we derive filters for universal noise by concatenating both filter types into a composite structure that filters both noise quadratures simultaneously, while still implementing a target qubit rotation. We refer to such constructions as universal Walsh modulated filters (UWMFs).

The general approach is conveniently illustrated through a particular concatenated structure obtained by embedding the $\operatorname{WPMF}_{1}^{(c)}(\theta) \equiv \operatorname{SK} 1(\theta)$ gate (Section 7) within the various segments of the $\mathrm{WAMF}_{0,3}^{(1)}$ gate (Section 6.1). The former is explicitly written

$$
\begin{gathered}
\mathrm{WPMF}_{1}^{(c)}(\theta) \equiv P_{2}\left[\begin{array}{cccc}
P_{1} & \tau_{l} & \theta_{l} & \phi_{l} \\
P_{0} & \tau_{\theta} & \theta & 0 \\
\Omega_{0} & \tau_{2 \pi} & 2 \pi & \phi_{\mathrm{SK} 1} \\
\Omega_{0} & \tau_{2 \pi} & 2 \pi & -\phi_{\mathrm{SK} 1}
\end{array}\right], \\
\Omega_{0}=\frac{\theta+4 \pi}{\tau_{\mathrm{SK} 1}}, \quad \tau_{\theta}=\frac{\theta}{\Omega_{0}}, \quad \phi_{\mathrm{SK} 1}(\theta) \equiv Y_{1}(\theta)=\cos ^{-1}\left(-\frac{\theta}{4 \pi}\right) .
\end{gathered}
$$

The basic concatenation procedure is now to replace each constant-amplitude pulse in $\mathrm{WAMF}_{0,3}^{(1)}$ with the constant-amplitude phase-modulated sequence implementing the equivalent rotation. Doing so effectively distributes the dephasing filter across the composite sequence, each subsequence of which filters amplitude noise. In our example, this takes place via the operator substitutions

$$
\begin{array}{ll}
P_{1}\left(X_{+} / 4,0\right) \rightarrow \mathrm{SK}^{(1)}\left(X_{+} / 4\right), & \tau_{\mathrm{SK} 1}^{(1)}=\tau / 4, \\
P_{2}\left(X_{-} / 2,0\right) \rightarrow \mathrm{SK}^{(2)}\left(X_{-} / 2\right), & \tau_{\mathrm{SK} 1}^{(2)}=\tau / 2, \\
P_{3}\left(X_{+} / 4,0\right) \rightarrow \mathrm{SK}^{(3)}\left(X_{+} / 4\right), & \tau_{\mathrm{SK} 1}^{(3)}=\tau / 4,
\end{array}
$$

where, as indicated, each SK1 is equal in duration to the original $\mathrm{WAMF}_{0,3}^{(1)}$ pulse being replaced. We denote this concatenated structure by $\mathrm{UWMF}_{1, \mathrm{SK} 1}$. The temporal profile is shown in Figure 7(a). The SK1 phase flips $\phi= \pm \phi_{\mathrm{SK} 1}$ are indicated by the oppositely oriented hatching within each constant-amplitude segment of the $\mathrm{WAMF}_{0,3}^{(1)}$ envelope; $\phi=0$ is indicated by white fill. Once again the total gate rotation is determined by Eq. (36) and, as in Figure 3, $X_{3}$ may be treated as an independent tuning parameter to achieve first-order 

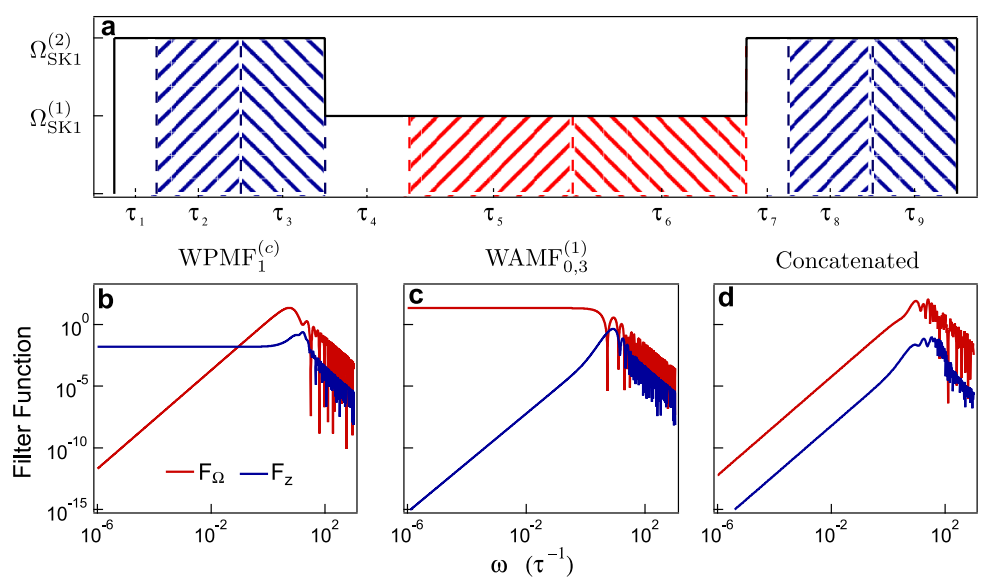

Figure 7 UWMFs. Concatenation scheme for universal noise suppression. (a) Concatenation of $\mathrm{WPMF}_{1}^{(c)} \equiv \mathrm{SK} 1$ within $\mathrm{WAMF}_{0,3}^{(1)}$ sequence yielding $\mathrm{UWMF}_{1, S K 1}$. White fill indicates rotations enacted with $\phi=0$; orientation of hatching denotes SK1 phase flips $\phi= \pm \phi_{\mathrm{SK} 1}$. (b) Filter-transfer functions for $\mathrm{WPMF}^{(c)} \equiv \mathrm{SK} 1$ sequence. (c) Filter-transfer functions for four-segment $\mathrm{WAMF}_{0,3}^{(1)}$ sequence. $(\mathbf{d})$ Filter-transfer functions for concatenated sequence.

filtering against dephasing noise. The dephasing and amplitude filter-transfer functions for the concatenated and tuned sequence (in this case for a net $\pi$ rotation) are shown in Figure 7(d), indicating effective filtering of both amplitude and dephasing noise. Below we detail two alternative constructions for realizing the UWMF structure.

\subsection{Concatenation Method 1: Constrain Sequencing of WAMF Envelope}

The amplitude-modulated envelope of the $\mathrm{WAMF}_{0,3}^{(1)}$ construction, as defined by Eq. (39), may be viewed as a sequence of 3 piecewise-constant rotations $\overrightarrow{\boldsymbol{\theta}}=\left(\frac{X_{+}}{4}, \frac{X_{-}}{2}, \frac{X_{+}}{4}\right)$, implemented over durations $\left(\frac{\tau}{4}, \frac{\tau}{2}, \frac{\tau}{4}\right)$ with Rabi rates $\overrightarrow{\boldsymbol{\Omega}}=\left(\frac{X_{+}}{\tau}, \frac{X_{-}}{\tau}, \frac{X_{+}}{\tau}\right)$. In our first concatenation method each rotation $\theta_{l}, l \in\{1,2,3\}$, is replaced by a constant-amplitude phase-modulated operation $\operatorname{SK} 1\left(\theta_{l}\right)$, implemented over a duration $\tau_{\mathrm{SK} 1}^{(l)}$ equal to that of the original rotation. That is, we constrain $\left(\tau_{\mathrm{SK} 1}^{(1)}, \tau_{\mathrm{SK} 1}^{(2)}, \tau_{\mathrm{SK} 1}^{(3)}\right)=\left(\frac{\tau}{4}, \frac{\tau}{2}, \frac{\tau}{4}\right)$. The constant Rabi rate $\Omega_{\mathrm{SK} 1}^{(l)}=\theta_{l} / \tau_{\mathrm{SK} 1}^{(l)}$ driving each SK1 sequence is thus given by

$$
\Omega_{\mathrm{SK} 1}^{(1)}=\Omega_{\mathrm{SK} 1}^{(3)}=\frac{1}{\tau}\left(X_{+}+16 \pi\right), \quad \Omega_{\mathrm{SK} 1}^{(2)}=\frac{1}{\tau}\left(X_{-}+8 \pi\right) .
$$

The composite structure may then be written

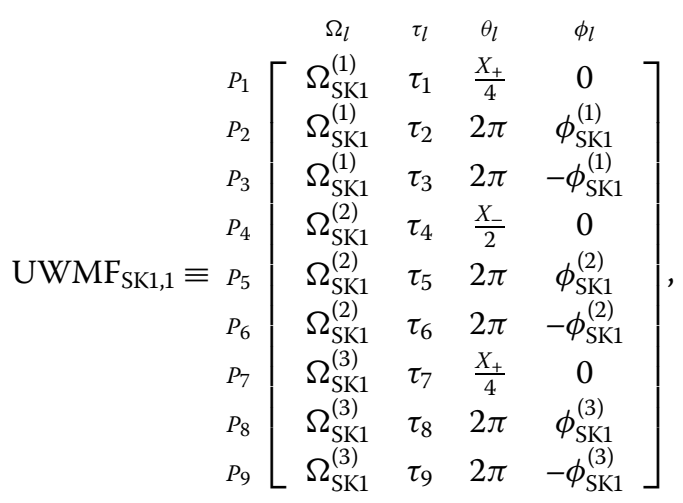




$$
\begin{aligned}
& \tau_{1,7}=\frac{\tau}{4}\left(\frac{X_{+}}{X_{+}+16 \pi}\right), \quad \tau_{2,3,8,9}=\tau\left(\frac{2 \pi}{X_{+}+16 \pi}\right), \\
& \tau_{4}=\frac{\tau}{2}\left(\frac{X_{-}}{X_{-}+8 \pi}\right), \quad \tau_{5,6}=\tau\left(\frac{2 \pi}{X_{-}+8 \pi}\right), \\
& \phi_{\mathrm{SK} 1}^{(1)}=\phi_{\mathrm{SK} 1}^{(3)}=\cos ^{-1}\left(-\frac{X_{+}}{16 \pi}\right), \quad \phi_{\mathrm{SK} 1}^{(2)}=\cos ^{-1}\left(-\frac{X_{-}}{8 \pi}\right) .
\end{aligned}
$$

Using this method one finds tunings of $X_{0}$ and $X_{3}$ such that both dephasing and amplitude noise are filtered to first-order, as in Figure 7 . These do not, however, correspond directly to the optimum Walsh coefficients found for simple $\mathrm{WAMF}_{0,3}^{(1)}$ construction shown in Figure 3(a). Rather, an equivalent tuning plot may be generated over the $X_{0} X_{3}$ domain, essentially identical to Figure 3 (a) but with minima shifted by a constant factor. The second method, detailed below, involves a slightly different construction in which we recover the original $\mathrm{WAMF}_{0,3}^{(1)}$ tuning plot.

\subsection{Concatenation Method 2: Constrain Sequencing of Target SK1 Rotations}

In the second construction we impose the constraint that $\tau_{1}: \tau_{4}: \tau_{7}=1: 2: 1$. That is, the target rotations within the three successive SK1 sequences follow the same timing sequence as the three constant-amplitude pulses being replaced, previously constituting the amplitude-modulated $\mathrm{WAMG}_{1}$ envelope. Thus we write $\left(\tau_{1}, \tau_{4}, \tau_{7}\right)=v(1,2,1)$ where $v$ is some fraction of the total duration $\tau$ of the composite structure to be determined.

Now, we know pulses $(1,4,7)$ execute the rotations $\left(\frac{X_{+}}{4}, \frac{X_{-}}{2}, \frac{X_{+}}{4}\right)$, given by the third row of Eq. (39). Hence the Rabi rates for these pulses in the composite structure must take the form $\left(\Omega_{1}, \Omega_{4}, \Omega_{7}\right)=\left(X_{+}, X_{-}, X_{+}\right) / 4 v$. However each SK1 sequence has constant Rabi rate and we therefore conclude

$$
\Omega_{\mathrm{SK} 1}^{(1)}=\Omega_{\mathrm{SK} 1}^{(3)}=\frac{1}{4 v} X_{+}, \quad \Omega_{\mathrm{SK} 1}^{(2)}=\frac{1}{4 v} X_{-} .
$$

Now, the duration of the composite sequence must satisfy

$$
\begin{aligned}
\tau & =\sum_{l=1}^{9} \frac{\theta_{l}}{\Omega_{1}} \\
& =2\left(\frac{2 \pi}{\Omega_{\mathrm{SK} 1}^{(1)}}\right)+2\left(\frac{2 \pi}{\Omega_{\mathrm{SK} 1}^{(2)}}\right)+2\left(\frac{2 \pi}{\Omega_{\mathrm{SK} 1}^{(3)}}\right)+4 v \\
& =4 \pi\left(\frac{1}{\Omega_{\mathrm{SK} 1}^{(1)}}+\frac{1}{\Omega_{\mathrm{SK} 1}^{(2)}}+\frac{1}{\Omega_{\mathrm{SK} 1}^{(3)}}\right)+4 v .
\end{aligned}
$$

Substituting in the results from Eq. (60) we therefore find $\tau=16 \pi v\left(2 / X_{+}+1 / X_{-}\right)+4 v$. Or, solving for $v$,

$$
\nu=\frac{1}{4} \tau(1+\pi \kappa)^{-1}, \quad \kappa:=4\left(\frac{2}{X_{+}}+\frac{1}{X_{-}}\right)
$$


concluding the construction. The composite structure may then be written

$$
\begin{aligned}
P_{1} & P_{2} \\
& P_{3} \\
P_{4} & \left.\begin{array}{cccc}
\Omega_{l} & \tau_{l} & \theta_{l} & \phi_{l} \\
\frac{X_{+}}{4 v} & v & \frac{X_{+}}{4} & 0 \\
\frac{X_{+}}{4 v} & \frac{8 \pi v}{X_{+}} & 2 \pi & \phi_{\mathrm{SK} 1}^{(1)} \\
\frac{X_{+}}{4 v} & \frac{8 \pi v}{X_{+}} & 2 \pi & -\phi_{\mathrm{SK} 1}^{(1)} \\
\frac{X_{-}}{4 v} & 2 v & \frac{X_{-}}{2} & 0 \\
\mathrm{UWMF}_{\mathrm{SK} 1,1} \equiv & P_{5} \\
X_{-} & \frac{8 \pi v}{X_{-}} & 2 \pi & \phi_{\mathrm{SK} 1}^{(2)} \\
P_{6} & P_{7} \\
\frac{X_{-}}{4 v} & \frac{8 \pi v}{X_{-}} & 2 \pi & -\phi_{\mathrm{SK} 1}^{(2)} \\
\frac{X_{+}}{4 v} & v & \frac{X_{+}}{4} & 0 \\
\frac{X_{+}}{4 v} & \frac{8 \pi v}{X_{+}} & 2 \pi & \phi_{\mathrm{SK} 1}^{(3)} \\
\frac{X_{+}}{4 v} & \frac{8 \pi v}{X_{+}} & 2 \pi & -\phi_{\mathrm{SK} 1}^{(3)}
\end{array}\right], \\
\phi_{\mathrm{SK} 1}^{(1)}=\phi_{\mathrm{SK} 1}^{(3)}=\cos ^{-1}\left(-\frac{X_{+}}{16 \pi}\right), & \phi_{\mathrm{SK} 1}^{(2)}=\cos ^{-1}\left(-\frac{X_{-}}{8 \pi}\right) .
\end{aligned}
$$

Minima of the cost function in the $X_{0} X_{3}$ domain for this construction are found in identical regions to those shown in Figure $3(\mathrm{a})$ for the simple $\mathrm{WAMF}_{0,3}^{(1)}$ construction. Substituting these minimizing values of $X_{0,3}$ into Eq. (65) thus optimizes the concatenated structure, yielding a desired net rotation (dictated by $X_{0}$ ) which filters both amplitude and dephasing noise to first order simultaneously.

\section{Effect of bandwidth limits on Walsh filters}

In the preceding sections of this paper filter design is based on optimizing the Walsh spectrum from which the relevant control structures are synthesized. This necessarily assumes perfectly square waveforms. Real control hardware, however, may suffer from bandwidth limitations which 'smooth out' the squareness of the pulse on the timescale of the application, leading to reduced filter performance. Here we show the assumption of perfect square pulses may be readily relaxed, with useful filter construction a simple matter of re-optimization in the Walsh-synthesis framework.

To illustrate the general procedure we consider the $\mathcal{M}$-segment WAMF. Each segment implements a rotation through angle $\theta_{l}=\Omega_{l} \tau_{l}$ over duration $\tau_{l}=\tau / \mathcal{M}$ and with constant Rabi rate $\Omega_{l}, l \in\{1, \ldots, \mathcal{M}\}$. The squareness of the resulting amplitude-modulated waveform may be relaxed by replacing the constant value $\Omega_{l}$ with an arbitrarily varying function of time in each segment.

In order to achieve this we consider Walsh synthesis over the rotation angle implemented in a single segment rather than the Rabi rate. That is, we write $\theta_{l}=\theta_{l}\left(X_{0}, X_{1}, \ldots, X_{N}\right)$ with the dependence on the Walsh spectra defined by the Hadamard-matrix equation

$$
\overrightarrow{\boldsymbol{\theta}}=\left(\theta_{1}, \theta_{2}, \ldots, \theta_{\mathcal{M}}\right)^{T}=(\tau / \mathcal{M}) H_{\mathcal{M}} \tilde{\mathbf{X}}
$$

Defined in this way, the $\mathcal{M}$-segment arbitrary-pulse sequence shares with the WAMF construction the property that the total gate rotation angle $\Theta=\sum_{l=1}^{n} \theta_{l}=X_{0} \tau$ is completely determined by the spectral amplitude of $\mathrm{PAL}_{0}$. The symmetry-based design rules similarly carry over and filter optimization proceeds in the same manner as for ordinary Walsh-modulated control by minimizing the filter cost function with respect to the Walsh spectrum. 
As a natural example of this approach we assume a Gaussian profile $G_{l}\left(t ; \mu_{l}, \sigma_{l}\right)$ defined on $t \in\left[t_{l-1}, t_{l}\right]$ with mean $\mu_{l}$ and standard deviation $\sigma_{l}$. Specifically, we construct

$$
G_{l}\left(t ; \mu_{l}, \sigma_{l}\right):=\frac{\theta_{l}}{C_{l} \sigma_{l} \sqrt{2 \pi}} \exp \left[-\frac{\left(t-\mu_{l}\right)^{2}}{2 \sigma_{l}^{2}}\right], \quad \mu_{l}=\frac{t_{l-1}+t_{l}}{2}, \sigma_{l}=g \tau / \mathcal{M}
$$

with $\mu_{l}$ the segment midpoint and $\sigma_{l}$ expressed as a multiple $g$ of the segment duration. The normalizing factor

$$
C_{l}:=\int_{t_{l-1}}^{t_{l}} \frac{1}{\sigma_{l} \sqrt{2 \pi}} \exp \left[-\frac{\left(t-\mu_{l}\right)^{2}}{2 \sigma_{l}^{2}}\right] d t
$$

is included to ensure the total rotation implemented by the Gaussian pulse in the lth segment is given by $\int_{t_{l}}^{t_{l-1}} G_{l}\left(t ; \mu_{l}, \sigma_{l}\right) d t=\theta_{l}$. We now impose the same structure on the segment rotations $\theta_{l}$ as done for WAMFs in the Walsh-synthesis framework, such that the smooth-pulse sequence remains strictly parametrized in the Walsh spectrum $\mathbf{X}$.

For concreteness, we examine the Gaussian-pulse variation on the 4-segment $\mathrm{WAMF}_{0,3}^{(1)}$ filter described by Eq. (39) for two different Gaussian profiles, illustrated in Figure 8(a), (c). The cost function $A_{z}\left(X_{3} ; X_{0}\right)=\int_{\omega_{L}}^{\omega_{c}} d \omega F_{z}\left(\omega \tau ; X_{3} ; X_{0}\right)$ may be computed by partitioning the time domain into a large number $N_{s}$ of subintervals on which the continuous Gaussian envelope is treated as approximately constant. Figure 8(b), (d) show a two-dimensional representation of $A_{z}\left(X_{3} ; X_{0}\right)$ integrated over the interval $\omega \in\left[10^{-9}, 10^{-6}\right] \tau^{-1}$. The value of $\log _{10}\left[A_{z}\left(X_{3} ; X_{0}\right)\right]$ is indicated by the color scale. Total sequence length is normalized to $\tau=1$ in this data, so the total gate rotation angle $\Theta \equiv X_{0}$ is given directly by the $X_{0}$-axis. Regions in blue represent effective (first-order) filter constructions, where the cost function is minimized.
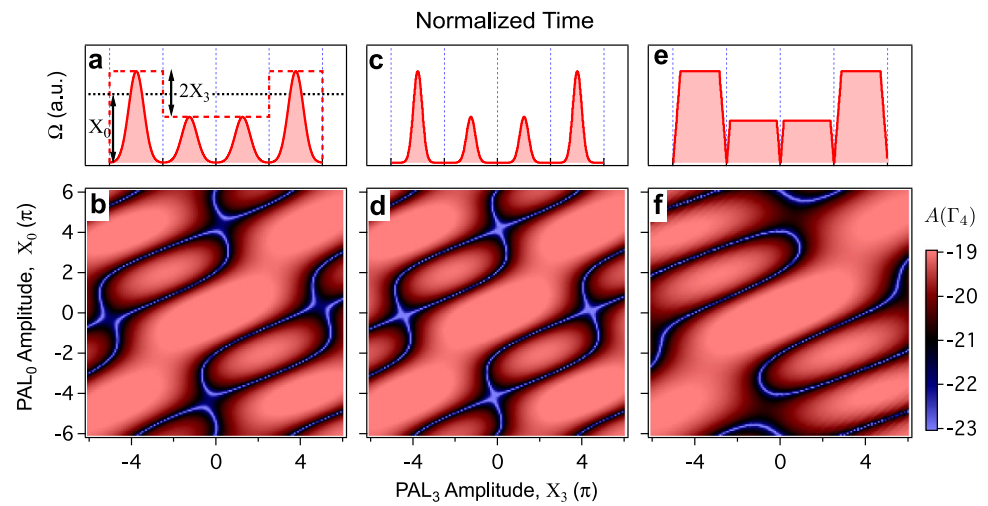

Figure 8 Shaped-pulse filter constructions. Construction of the first-order Walsh amplitude modulated dephasing-suppressing filter using shaped pulse segments. (a), (c), (e) Schematic representation of Walsh synthesis for a four segment gate of discrete Gaussian or trapezoidal segments. Walsh synthesis determines the overall amplitude of individual pulses with fixed duration and standard deviation, setting the effective pulse area in each segment. The metric $g$ takes value 1/6 in panel (a), and 1/12 in panel (c). (e) Trapezoidal pulses are characterized by a constant slope such that all angles are a fraction of a square waveform defined as $F \frac{\pi}{2}$. Here $F=0.992$. In all pulse constructions the pulse profile is computed over 100 discrete time steps, permitting calculation of relevant filter-transfer functions. (b), (d), (f) Two-dimensional representation of the integral metric defining our target cost function, $A\left(\boldsymbol{\Gamma}_{4}\right)$ integrated over the stopband $\omega \in\left[10^{-9}, 10^{-6}\right] \tau^{-1}$ for the corresponding pulse forms above. Areas in blue minimize $A\left(\boldsymbol{\Gamma}_{4}\right)$, representing effective filter constructions. The $X_{0}$ determines the net rotation enacted in a gate while $X_{3}$ determines the modulation depth, as represented in (a). 
Comparing with Figure 3 we conclude useful filter construction using Gaussian pulses is a simple matter of re-optimization in the Walsh-synthesis framework. This is readily achieved using a Nelder-Mead optimization of $A_{z}\left(X_{3} ; X_{0}\right)$ for any particular choice of $g$, $\omega_{L}, \omega_{c}, X_{0}$ or $N_{s}$. Minor changes in the filter performance and optimal constructions arise with changes in Gaussian pulse parameters such as $g$. Comparison with pulses constructed using a trapezoidal form (Figure 8(e)) we find a different optimization outcome that more closely approximates standard square pulses. Nonetheless, these results show that, irrespective the specific pulse form, re-optimization over the Walsh coefficients remains a direct method to construct useful filters. In cases where unknown waveform distortion is likely in hardware, it is possible to implement automated feedback mechanisms, as has previously been demonstrated in dynamical decoupling experiments [15].

We may also explore the impact of finite modulation bandwidth on the application of square pulses if re-optimization of the waveform is, for some reason, not possible. In order to explore these effects we systematically relax the infinite-modulation-bandwidth assumption underlying any square-pulse approximation by processing the ideal timedomain profile through a bandlimited digital filter with a user-defined cutoff. This results in an imperfect (bandlimited) profile envelope, effectively due to a reconstruction based on a truncated Fourier series. These profile distortions, manifesting as implementation errors, reduce filter performance, quantified by an increase in the area under the corresponding filter-transfer function.

In this example we again consider the amplitude-modulated profile associated with the $\operatorname{WAMF}_{0,3}^{(1)}$ gate $\left(X_{0}=3 \pi, X_{3}=\pi\right)$, and impose band limitations using a digital firstorder Butterworth filter. Here the time domain is partitioned into $1 / f_{s}$ subintervals, where $f_{s}=1 / 2^{11}$ is the sampling rate of the digital filter. The bandlimited envelope of reconstructed waveform is then a function of the Butterworth cutoff frequency $f_{c}$. As the cutoff approaches the Nyquist frequency $f_{s} / 2$ (the maximum possible value), the bandlimited effects are reduced and the reconstructed waveform approaches the ideal square-pulse envelope. Figure $9\left(\right.$ a) illustrates this as we increase $f_{c} / f_{s}$. The corresponding filter characteristics are shown in Figure 9(c) where we plot $F_{z}(\omega)$ as a function of $f_{c} / f_{s}$. To compute these filter-transfer functions we treat the reconstructed waveforms as amplitudemodulated sequences consisting of $n=1 / f_{s}$ segments whose Rabi-rates are determined by the bandlimited envelope (see Figure 9(b)). The solid black traces in Figure 9(a), (b), (c) respectively show the ideal profile and corresponding filter-transfer function, against which the filter performance of the bandlimited gates are benchmarked. As we decrease the cut-

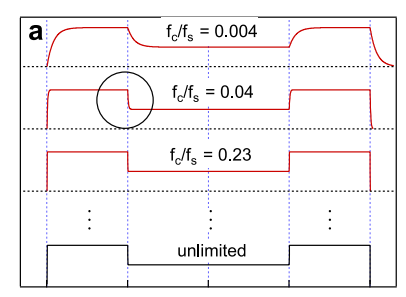

Gate Time

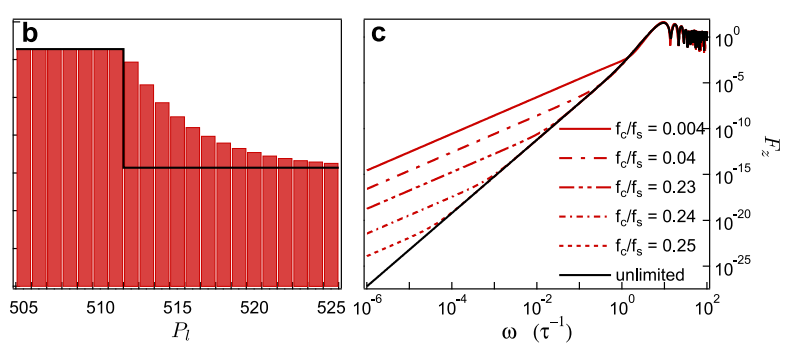

Figure 9 Bandlimited filters. Effect of bandwidth limits on Walsh filters. (a) Bandlimited reconstructions of WAMF $_{0,3}^{(1)}$ modulation envelope as a function of first-order Butterworth filter parameter $f_{c} / f_{s}$. (b) Red: Closeup of circled region in (a); Black ideal square profile. (c) Filter-transfer functions for profiles in (a). 
off the integrated area under $F_{z}(\omega)$ gradually increases. However as this manifests first in the low-frequency region, even these bandlimited gates can provide useful filtering, given sufficient hardware precision. Similarly motivated studies in dynamic decoupling show analogous low-frequency filter performance decay with pulse-timing errors.

\section{Conclusion}

As the size and complexity of quantum information processing technologies increase, resource-efficiency will play a vital role in selecting methods designed to reduce errors in quantum coherent hardware systems. The pressure to minimize quantum-hardware overhead is likely to make open-loop control protocols a key element in the design of errorrobust quantum information systems [14]. For these to be practically useful, however, it is important to move toward realistic control and noise models.

Decoherence in real driven systems is predominantly due to low-frequency correlated noise environments. This strongly motivates our study of bounded-strength control as a noise filtering problem using time-dependent, non-Markovian error models. Moreover, in contrast with traditional DD schemes, the added complications of treating boundedstrength control - due to the continual presence of noise interactions during control operations and the resulting nonlinear dynamics - necessitates a streamlined approach to the design of noise-filtering control. The generalized filter-transfer function framework we employ takes as input experimentally measurable characteristics of the environment namely noise power spectra - and provides a simple framework for both control construction and the calculation of predicted operational fidelities. It also efficiently captures the control nonlinearities implicit in situations where control and noise Hamiltonians do not commute. We have exploited these strengths to pursue a simple variational procedure for filter design by minimizing a cost function over the relevant control space.

A key strength of the method we have presented is derived from our use of functional analysis for the crafting of effective noise-filtering control protocols. In particular, employing the Walsh basis brings an intuitive set of analytic design rules for filter construction that further constrain the possible filter-construction space [51]. For instance, a userimposed limit on the acceptable number of pulse segments in a filter construction impose additional constraints due to Walsh-function symmetry, spectral properties, and the level of recursiveness of the Walsh functions (measured by the Hamming weight of the Walsh Paley-ordered index).

In addition to efficiency of synthesis is the intrinsic compatibility with hardware controllers that comes with the selection of Walsh functions as our basis set. This is particularly important in the layered architecture for quantum information systems mentioned throughout this paper. In such a setting, it rapidly becomes undesirable to mandate a significant amount of communication between the physical qubit layer and hardware at the highest levels of system abstraction. This suggests that controllers implementing dynamic error suppression protocols (here producing noise filters for arbitrary driven operations) should be reasonably simple to implement in standard digital hardware and should require only limited communication bandwidth to higher levels of the system.

These considerations are explicitly met in crafting control solutions from the Walsh basis. First, the Walsh functions are defined using integer multiples of a fundamental clock period, meaning that limitations of finite timing precision in the definition of a control protocol are automatically inbuilt. Further, given a particular Walsh-modulated control 
protocol is entirely defined by its Walsh spectrum, programming of the controller can in principle be reduced to a simple vector of numbers representing the Walsh spectrum and minimum timestep. All other information e.g. the total time, total number of timesteps, etc., is carried implicitly in the spectrum. Moreover, the actual Walsh-function generation is compatible with simple hardware systems (adding of various harmonics of a fundamental square-wave clock) and when Walsh synthesis is performed at the level of the controller hardware, this may provide a path to on-the-fly synthesis of the required modulation waveform. Such capabilities also reduce the complexity of running automated hardware-driven optimization procedures for finding relevant control waveforms [15], by allowing efficient generation of many trial waveforms without the need for large memory stores at the local controller.

Synthesizing all of these considerations the Walsh-modulated noise filters we have developed in this work provide one of the first solutions for error suppression at the physicalqubit level simultaneously meeting the physical and engineering requirements we outline above for scalable control solutions. Using this framework we have derived a range of novel filters, chiefly WAMFs for dephasing noise and WPMFs for amplitude noise. Both are capable of spectral optimization subject to physically motivated constraints such as implementing target qubit rotations. These design forms are also compatible with concatenation for filtering universal noise. Interestingly, our approach unifies a number of existing composite pulse sequencing schemes; we have revealed how Walsh-modulated filter construction naturally incorporates familiar sequences (e.g., DCG, SK1, P2, BB1) in a non-Markovian time-dependent noise context. This potential to incorporate other approaches may prove useful in building a consistent picture of the scope and applicability of the many and varied schemes that continue to be developed by the quantum control community. These considerations make the Walsh basis an attractive design platform and we believe this simple framework will provide a straightforward path for the development of improved quantum control techniques.

\section{Appendix A: Detailed filter-transfer function derivation}

In this appendix we derive the computational form of the first-order infidelity $\left\langle a_{1}^{2}\right\rangle=$ $\left\langle\mathbf{a}_{1} \mathbf{a}_{1}^{T}\right\rangle$ expressed in Eq. (4). Recall, we write the total evolution operator $U(t)=U_{c}(t) \tilde{U}(t)$ where the error propagator $\tilde{U}(t)$ satisfies the Schrodinger equation $i \dot{\tilde{U}}(t)=\tilde{H}_{0}(t) \tilde{U}(t)$ in a frame co-rotating with the control, defined by the toggling frame Hamiltonian $\tilde{H}_{0}(t):=$ $U_{c}^{\dagger}(t) H_{0}(t) U_{c}(t)$. We may obtain an arbitrarily accurate, unitary estimate of the error propagator by performing a Magnus expansion, whereby $\tilde{U}(\tau)=\exp [-i \Phi(\tau)]$ and the effective error operator $\Phi(\tau)=\sum_{\mu=1}^{\infty} \Phi_{\mu}(\tau)$ at the end of the interaction has Magnus expansion terms

$$
\begin{aligned}
\Phi_{1}(\tau)= & \int_{0}^{\tau} d t \tilde{H}_{0}(t), \\
\Phi_{2}(\tau)= & -\frac{i}{2} \int_{0}^{\tau} d t_{1} \int_{0}^{t_{1}} d t_{2}\left[\tilde{H}_{0}\left(t_{1}\right), \tilde{H}_{0}\left(t_{2}\right)\right], \\
\Phi_{3}(\tau)= & \frac{1}{6} \int_{0}^{\tau} d t_{1} \int_{0}^{t_{1}} d t_{2} \int_{0}^{t_{2}} d t_{3}\left\{\left[\tilde{H}_{0}\left(t_{1}\right),\left[\tilde{H}_{0}\left(t_{2}\right), \tilde{H}_{0}\left(t_{3}\right)\right]\right]\right. \\
& \left.+\left[\tilde{H}_{0}\left(t_{3}\right),\left[\tilde{H}_{0}\left(t_{2}\right), \tilde{H}_{0}\left(t_{1}\right)\right]\right]\right\},
\end{aligned}
$$


These generally take the form of time-ordered integrals over nested commutators in $\tilde{H}_{0}(t)$.

We define the error vector $\mathbf{a}(\tau)$ by re-expressing the operator $\Phi(\tau) \equiv \mathbf{a}(\tau) \cdot \boldsymbol{\sigma}$ in the basis of Pauli operators. ${ }^{\mathrm{m}}$ Assuming unitary processes, one may then employ vector identities to expand $\mathbf{a}(\tau)=\sum_{\mu=1}^{\infty} \mathbf{a}_{\mu}(\tau)$ in an infinite power series such that $\Phi_{\mu}(\tau)=\mathbf{a}_{\mu}(\tau) \cdot \sigma, \forall \mu \in \mathbb{N}$ [49]. The control propagator is therefore written $\tilde{U}(\tau)=\exp \left[-i \sum_{\mu=1}^{\infty} \mathbf{a}_{\mu}(\tau) \cdot \boldsymbol{\sigma}\right]$ and may be approximated, with arbitrary accuracy, as a unitary operator in simple exponential form. In this paper we consider the first-order approximation $\mathbf{a}(\tau) \approx \mathbf{a}_{1}(\tau)$. Hence we restrict attention to deriving the form of $\mathbf{a}_{1}(\tau)$ which, using Eq. (70), satisfies

$$
\mathbf{a}_{1}(\tau) \cdot \sigma=\Phi_{1}(\tau)=\int_{0}^{\tau} d t \tilde{H}_{0}(t)
$$

Our first task is therefore to derive a computationally useful forms for the toggling frame Hamiltonian $\tilde{H}_{0}(t)$. This is done in the following section.

\section{Toggling frame Hamiltonian $\tilde{H}_{0}(t)$ : computational form}

The noise Hamiltonian $H_{0}(t)=H_{0}^{(z)}(t)+H_{0}^{(\Omega)}(t)$, presented in Section 2 is linear in the dephasing and amplitude contributions. Since the $\tilde{H}_{0}(t)$ is linear in $H_{0}(t)$ we may write

$$
\tilde{H}_{0}(t)=\tilde{H}_{0}^{(z)}(t)+\tilde{H}_{0}^{(\Omega)}(t)
$$

where we have defined the dephasing and amplitude toggling frame Hamiltonians by

$$
\begin{aligned}
& \tilde{H}_{0}^{(z)}(t):=U_{c}^{\dagger}(t) H_{0}^{(z)}(t) U_{c}(t), \\
& \tilde{H}_{0}^{(\Omega)}(t):=U_{c}^{\dagger}(t) H_{0}^{(\Omega)}(t) U_{c}(t) .
\end{aligned}
$$

It is convenient to employ the definitions of the scalar functions $R_{i j}(t), R_{i j}^{P_{l}}\left(t-t_{l-1}\right)$ and $\Lambda_{i j}^{(l-1)}, i, j \in\{x, y, z\}$ introduced by Green et. al [49]. These are defined as the Cartesian expansion coefficients, in the basis of Pauli matrices, of the following operators

$$
\begin{aligned}
& U_{c}^{\dagger}(t) \hat{\sigma}_{i} U_{c}(t)=\sum_{j=x, y, z} R_{i j}(t) \hat{\sigma}_{j}, \\
& U_{c}^{\dagger}\left(t, t_{l-1}\right) \hat{\sigma}_{i} U_{c}\left(t, t_{l-1}\right)=\sum_{j=x, y, z} R_{i j}^{P_{l}}\left(t-t_{l-1}\right) \hat{\sigma}_{j}, \\
& Q_{l-1}^{\dagger} \hat{\sigma}_{i} Q_{l-1}=\sum_{j=x, y, z} \Lambda_{i j}^{(l-1)} \hat{\sigma}_{j} .
\end{aligned}
$$

These functions then serve as matrix elements, defining the computational matrices

$$
\begin{array}{ll}
\text { Total Control Matrix : } & {[\mathbf{R}(t)]_{i j}:=\frac{1}{2} \operatorname{Tr}\left[U_{c}^{\dagger}(t) \hat{\sigma}_{i} U_{c}(t) \hat{\sigma}_{j}\right],} \\
\text { Local Control Matrix : } & {\left[\mathbf{R}^{P_{l}}\left(t-t_{l-1}\right)\right]_{i j}:=\frac{1}{2} \operatorname{Tr}\left[U_{c}^{\dagger}\left(t, t_{l-1}\right) \hat{\sigma}_{i} U_{c}\left(t, t_{l-1}\right) \hat{\sigma}_{j}\right],} \\
\text { Control History Matrix }: & {\left[\boldsymbol{\Lambda}^{(l-1)}\right]_{i j}:=\frac{1}{2} \operatorname{Tr}\left[Q_{l-1}^{\dagger} \hat{\sigma}_{i} Q_{l-1} \hat{\sigma}_{j}\right],}
\end{array}
$$

where the above expressions follow from post-multiplying Eqs. (75), (76) and (77) by $\hat{\sigma}_{j}$, taking the trace and using the linearity of the trace operation. The matrix $\mathbf{R}(t)$ captures the 
qubit dynamics, in the time-domain, due to the control Hamiltonian at any time; $\mathbf{R}^{P l}(t-$ $t_{l-1}$ ) captures essentially the same information, but restricted to the time interval $t \in I_{l}$. That is, during the $l$ th pulse. The $3 \times 3$ Control History Matrix $\Lambda^{(l-1)}$, on the other hand, captures the accumulated effect of the previous $l-1$ completed pulses, implemented via the cumulative operator $Q_{l-1}$.

\section{Dephasing toggling Hamiltonian $\tilde{H}_{0}^{(z)}(t)$}

Substituting Eq. (2) into Eq. (73) the dephasing noise component of $\tilde{H}_{0}(t)$ takes the form

$$
\tilde{H}_{0}^{(z)}(t)=\beta_{z}(t) U_{c}^{\dagger}(t) \hat{\sigma}_{z} U_{c}(t)
$$

Using Eq. (75), we may express $U_{c}^{\dagger}(t) \hat{\sigma}_{z} U_{c}(t)=\sum_{j} R_{z j}(t) \hat{\sigma}_{j}, j \in\{x, y, z\}$, yielding

$$
\tilde{H}_{0}^{(z)}(t)=\beta_{z}(t) \mathbf{R}^{(z)}(t) \boldsymbol{\sigma},
$$

where the time-domain Dephasing Control Vector $\mathbf{R}^{(z)}(t)$ is defined as the third row of $\mathbf{R}(t)$. Here, and in the following derivations, for notational simplicity $\sigma$ is understood to be the column vector

$$
\sigma \equiv\left(\hat{\sigma}_{x}, \hat{\sigma}_{y}, \hat{\sigma}_{z}\right)^{T}
$$

The computational form of $\mathbf{R}^{(z)}(t)$, by inspection of the more general computational form for $\mathbf{R}(t)$ derived in Appendix B, is

$$
\mathbf{R}^{(z)}(t)=\sum_{l=1}^{n} G^{(l)}(t) \mathbf{R}_{z}^{P_{l}}\left(t-t_{l-1}\right) \mathbf{\Lambda}^{(l-1)} .
$$

\section{Amplitude toggling Hamiltonian $\tilde{H}_{0}^{(\Omega)}(t)$}

Similarly, substituting Eq. (3) into Eq. (74) we find

$$
\begin{aligned}
\tilde{H}_{0}^{(\Omega)}(t) & =\beta_{\Omega}(t) \sum_{l=1}^{n} G^{(l)}(t) \frac{\Omega_{l}}{2}\left\{U_{c}^{\dagger}(t) \hat{\sigma}_{\phi_{l}} U_{c}(t)\right\} \\
& =\beta_{\Omega}(t) \sum_{l=1}^{n} G^{(l)}(t) \frac{\Omega_{l}}{2} Q_{l-1}^{\dagger} U_{c}^{\dagger}\left(t, t_{l-1}\right) \hat{\sigma}_{\phi_{l}} U_{c}\left(t, t_{l-1}\right) Q_{l-1},
\end{aligned}
$$

where in the second line we have substituted $U_{c}(t)=\sum_{l=1}^{n} G^{(l)}(t) U_{c}\left(t, t_{l-1}\right) Q_{l-1}$ using Eq. (10). From Eq. (8) the control operator $U_{c}\left(t, t_{l-1}\right)=\exp \left[-i \frac{\Omega_{l}}{2} \hat{\sigma}_{\phi_{l}}\left(t-t_{l-1}\right)\right]$ commutes with $\hat{\sigma}_{\phi l}, \forall l \in\{1, \ldots, n\}$. That is, coaxial amplitude noise always 'tracks' the direction of control. Hence Eq. (86) reduces to

$$
\tilde{H}_{0}^{(\Omega)}(t)=\beta_{\Omega}(t) \sum_{l=1}^{n} G^{(l)}(t) \frac{\Omega_{l}}{2} Q_{l-1}^{\dagger} \hat{\sigma}_{\phi_{l}} Q_{l-1} .
$$

Now, from Eq. (77), we know $Q_{l-1}^{\dagger} \hat{\sigma}_{i} Q_{l-1}=\Lambda_{i}^{(l-1)} \boldsymbol{\sigma}$, where $\boldsymbol{\Lambda}_{i}^{(l-1)}$ denotes the $i$ th row of $\Lambda^{(l-1)}$. We may therefore write

$$
\frac{\Omega_{l}}{2} Q_{l-1}^{\dagger}, \hat{\sigma}_{\phi_{l}} Q_{l-1}=\frac{\Omega_{l}}{2}\left[\cos \left(\phi_{l}\right) \Lambda_{x}^{(l-1)}+\sin \left(\phi_{l}\right) \boldsymbol{\Lambda}_{y}^{(l-1)}\right]=\overrightarrow{\mathbb{T}}^{(l)} \boldsymbol{\Lambda}^{(l-1)} \boldsymbol{\sigma}
$$


where $\vec{T}^{(l)} \equiv \frac{\Omega_{l}}{2}\left(\cos \left(\phi_{l}\right), \sin \left(\phi_{l}\right), 0\right)$ is the Projection Vector defined in Eq. (18). Consequently, substituting into Eq. (87), the amplitude toggling Hamiltonian is re-expressed

$$
\tilde{H}_{0}^{(\Omega)}(t)=\beta_{\Omega}(t) \mathbf{R}^{(\Omega)}(t) \boldsymbol{\sigma}
$$

where, for compactness, we have defined the time-domain Amplitude Control Vector

$$
\mathbf{R}^{(\Omega)}(t):=\sum_{l=1}^{n} G^{(l)}(t) \overrightarrow{\mathbb{T}}^{(l)} \boldsymbol{\Lambda}^{(l-1)}
$$

\section{First-order error vector}

To summarize of the previous sections, the error propagator is now written

$$
\begin{aligned}
\tilde{U}(\tau) & =\exp [-i \Phi(\tau)] \\
& \approx \exp \left[-i \mathbf{a}_{1}(\tau) \boldsymbol{\sigma}\right] \\
& =\exp \left[-i \int_{0}^{\tau} d t \tilde{H}_{0}(t)\right] \\
& =\exp \left[-i\left(\int_{0}^{\tau} d t \tilde{H}_{0}^{(z)}(t)+\int_{0}^{\tau} d t \tilde{H}_{0}^{(\Omega)}(t)\right)\right] \\
& =\exp \left[-i\left(\int_{0}^{\tau} d t \beta_{z}(t) \mathbf{R}^{(z)}(t)+\int_{0}^{\tau} d t \beta_{\Omega}(t) \mathbf{R}^{(\Omega)}(t)\right) \boldsymbol{\sigma}\right] .
\end{aligned}
$$

The first-order error vector consequently takes the form

$$
\begin{aligned}
\mathbf{a}_{1}(\tau) & =\int_{0}^{\tau} d t \beta_{z}(t) \mathbf{R}^{(z)}(t)+\int_{0}^{\tau} d t \beta_{\Omega}(t) \mathbf{R}^{(\Omega)}(t) \\
& =\mathbf{a}_{1}^{(z)}+\mathbf{a}_{1}^{(\Omega)},
\end{aligned}
$$

where we define components

$$
\begin{aligned}
& \mathbf{a}_{1}^{(z)}(\tau):=\int_{0}^{\tau} d t \beta_{z}(t) \mathbf{R}^{(z)}(t) \quad \text { (Dephasing Error Vector) }, \\
& \mathbf{a}_{1}^{(\Omega)}(\tau):=\int_{0}^{\tau} d t \beta_{\Omega}(t) \mathbf{R}^{(\Omega)}(t) \quad(\text { Amplitude Error Vector }) .
\end{aligned}
$$

\section{First-order infidelity}

The time-domain representation of the first-order infidelity $\left\langle a_{1}^{2}\right\rangle=\left\langle\mathbf{a}_{1} \mathbf{a}_{1}^{T}\right\rangle$ now follows directly from Eq. (96). For filtering time-dependent noise, however, it is more useful to transform to a spectral representation in which case $\left\langle a_{1}^{2}\right\rangle$ separates into dephasing and amplitude noise terms, each appearing as an overlap integral between the noise PSD and a frequency-domain filter-transfer function. In this section we summarize this derivation, and define the filter-transfer functions. In the following section we present the final forms for these filter-transfer functions.

The modulus square of the first-order error vector, using Eq. (96), is given by

$$
\begin{aligned}
\mathbf{a}_{1} \mathbf{a}_{1}^{T} & =\left(\mathbf{a}_{1}^{(z)}+\mathbf{a}_{1}^{(\Omega)}\right)\left(\mathbf{a}_{1}^{(z)}+\mathbf{a}_{1}^{(\Omega)}\right)^{T} \\
& =\mathbf{a}_{1}^{(z)} \mathbf{a}_{1}^{(z) T}+\mathbf{a}_{1}^{(z)} \mathbf{a}_{1}^{(\Omega) T}+\mathbf{a}_{1}^{(\Omega)} \mathbf{a}_{1}^{(z) T}+\mathbf{a}_{1}^{(\Omega)} \mathbf{a}_{1}^{(\Omega) T} .
\end{aligned}
$$


Substituting Eqs. (98) and (99) and taking the ensemble average over time, the first-order infidelity $\left\langle a_{1}^{2}\right\rangle=\left\langle\mathbf{a}_{1} \mathbf{a}_{1}^{T}\right\rangle$ takes the form

$$
\begin{aligned}
\left\langle a_{1}^{2}\right\rangle= & \int_{0}^{\tau} d t_{1} \int_{0}^{\tau} d t_{2}\left\langle\beta_{\Omega}\left(t_{1}\right) \beta_{\Omega}\left(t_{2}\right)\right| \mathbf{R}^{(\Omega)}\left(t_{1}\right)\left[\mathbf{R}^{(\Omega)}\left(t_{2}\right)\right]^{T} \\
& +\int_{0}^{\tau} d t_{1} \int_{0}^{\tau} d t_{4}\left\langle\beta_{\Omega}\left(t_{1}\right) \beta_{z}\left(t_{4}\right)\right\rangle \mathbf{R}^{(\Omega)}\left(t_{1}\right)\left[\mathbf{R}^{(z)}\left(t_{4}\right)\right]^{T} \\
& +\int_{0}^{\tau} d t_{3} \int_{0}^{\tau} d t_{2}\left\langle\beta_{z}\left(t_{3}\right) \beta_{\Omega}\left(t_{2}\right)\right\rangle \mathbf{R}^{(z)}\left(t_{3}\right)\left[\mathbf{R}^{(\Omega)}\left(t_{2}\right)\right]^{T} \\
& +\int_{0}^{\tau} d t_{3} \int_{0}^{\tau} d t_{4}\left\langle\beta_{z}\left(t_{3}\right) \beta_{z}\left(t_{4}\right)\right| \mathbf{R}^{(z)}\left(t_{3}\right)\left[\mathbf{R}^{(z)}\left(t_{4}\right)\right]^{T} .
\end{aligned}
$$

Here the time average only operates on the noise fields, not on the control vectors since these are deterministic. Assuming, as in Section 2, $\beta_{\Omega}(t)$ and $\beta_{z}(t)$ are uncorrelated, classical random variables with zero mean, the two-point cross-correlation functions $\left\langle\beta_{\Omega}\left(t_{i}\right) \beta_{z}\left(t_{j}\right)\right\rangle=\left\langle\beta_{z}\left(t_{k}\right) \beta_{\Omega}\left(t_{l}\right)\right\rangle=0$. Hence the infidelity reduces to

$$
\begin{aligned}
\left\langle a_{1}^{2}\right\rangle= & \int_{0}^{\tau} d t_{1} \int_{0}^{\tau} d t_{2}\left\langle\beta_{\Omega}\left(t_{1}\right) \beta_{\Omega}\left(t_{2}\right)\right| \mathbf{R}^{(\Omega)}\left(t_{1}\right)\left[\mathbf{R}^{(\Omega)}\left(t_{2}\right)\right]^{T} \\
& +\int_{0}^{\tau} d t_{3} \int_{0}^{\tau} d t_{4}\left\langle\beta_{z}\left(t_{3}\right) \beta_{z}\left(t_{4}\right)\right| \mathbf{R}^{(z)}\left(t_{3}\right)\left[\mathbf{R}^{(z)}\left(t_{4}\right)\right]^{T} .
\end{aligned}
$$

We further assuming wide-sense-stationary, so the remaining two-point correlation functions depend only on the time difference, and therefore reduce to auto-correlation functions. In this case we may invoke the Wiener-Khinchin theorem [77],

$$
\left\langle\beta\left(t_{1}\right) \beta\left(t_{2}\right)\right\rangle=\frac{1}{2 \pi} \int_{-\infty}^{\infty} S_{\beta}(\omega) e^{i \omega\left(t_{2}-t_{1}\right)} d \omega
$$

which relates the autocorrelation function of a signal $\beta(t)$ to the Fourier transform of its $\operatorname{PSD} S_{\beta}(\omega)$. Denoting the dephasing and amplitude noise PSDs by $S_{z}(\omega)$ and $S_{\Omega}(\omega)$, we may therefore re-express $\left\langle a_{1}^{2}\right\rangle$ in terms of the noise spectral properties, yielding

$$
\begin{aligned}
\left\langle a_{1}^{2}\right\rangle= & \frac{1}{2 \pi} \int_{-\infty}^{\infty} d \omega S_{\Omega}(\omega)\left[\int_{0}^{\tau} d t_{1} e^{-i \omega t_{1}} \mathbf{R}^{(\Omega)}\left(t_{1}\right) \int_{0}^{\tau} d t_{2} e^{i \omega t_{2}}\left[\mathbf{R}^{(\Omega)}\left(t_{2}\right)\right]^{T}\right] \\
& +\frac{1}{2 \pi} \int_{-\infty}^{\infty} d \omega^{\prime} S_{z}\left(\omega^{\prime}\right)\left[\int_{0}^{\tau} d t_{3} e^{-i \omega^{\prime} t_{3}} \mathbf{R}^{(z)}\left(t_{3}\right) \int_{0}^{\tau} d t_{4} e^{i \omega^{\prime} t_{4}}\left[\mathbf{R}^{(z)}\left(t_{4}\right)\right]^{T}\right] .
\end{aligned}
$$

Defining the frequency-domain control vectors via the integral transforms

$$
\mathbf{R}^{(\Omega)}(\omega)=-i \omega \int_{0}^{\tau} d t e^{i \omega t} \mathbf{R}^{(\Omega)}(t), \quad \mathbf{R}^{(z)}(\omega)=-i \omega \int_{0}^{\tau} d t e^{i \omega t} \mathbf{R}^{(z)}(t)
$$

it is then straightforward to further re-express the infidelity in terms the spectral properties of the control, yielding

$$
\begin{aligned}
\left\langle a_{1}^{2}\right\rangle= & \frac{1}{2 \pi} \int_{-\infty}^{\infty} \frac{d \omega}{\omega^{2}} S_{\Omega}(\omega)\left[\mathbf{R}^{(\Omega)}(\omega)\right]^{*}\left[\mathbf{R}^{(\Omega)}(\omega)\right]^{T} \\
& +\frac{1}{2 \pi} \int_{-\infty}^{\infty} \frac{d \omega^{\prime}}{\omega^{\prime 2}} S_{z}\left(\omega^{\prime}\right)\left[\mathbf{R}^{(z)}\left(\omega^{\prime}\right)\right]^{*}\left[\mathbf{R}^{(z)}\left(\omega^{\prime}\right)\right]^{T} .
\end{aligned}
$$


Defining the frequency-domain filter-transfer functions,

$$
\begin{aligned}
& F_{z}(\omega):=\left[\mathbf{R}^{(z)}(\omega)\right]^{*}\left[\mathbf{R}^{(z)}(\omega)\right]^{T} \quad \text { (Dephasing Filter-Transfer Function), } \\
& F_{\Omega}(\omega):=\left[\mathbf{R}^{(\Omega)}(\omega)\right]^{*}\left[\mathbf{R}^{(\Omega)}(\omega)\right]^{T} \quad \text { (Amplitude Filter-Transfer Function). }
\end{aligned}
$$

we therefore recover Eq. (4)

$$
\left\langle a_{1}^{2}\right\rangle=\frac{1}{2 \pi} \int_{-\infty}^{\infty} \frac{d \omega}{\omega^{2}} S_{z}(\omega) F_{z}(\omega)+\frac{1}{2 \pi} \int_{-\infty}^{\infty} \frac{d \omega^{\prime}}{\omega^{\prime 2}} S_{\Omega}\left(\omega^{\prime}\right) F_{\Omega}\left(\omega^{\prime}\right) .
$$

\section{Appendix B: Control vectors: computational forms}

The dephasing and amplitude filter-transfer functions $F_{z}(\omega)$ and $F_{\Omega}(\omega)$ are obtained by taking the modulus square respectively of the frequency-domain dephasing and amplitude control vectors $\mathbf{R}^{(z)}(\omega)$ and $\mathbf{R}^{(\Omega)}(\omega)$, defined by Eq. (111) in terms of a Fourier-type transform. In this section we derive the computationally useful forms of $\mathbf{R}^{(z)}(\omega)$ and $\mathbf{R}^{(\Omega)}(\omega)$.

\section{Total Control Matrix $\mathbf{R}(t)$ computational form}

The time-domain Total Control Matrix $\mathbf{R}(t)$ is defined by Eq. (78) with elements $R_{i j}(t):=$ $\frac{1}{2} \operatorname{Tr}\left[U_{c}^{\dagger}(t) \hat{\sigma}_{i} U_{c}(t) \hat{\sigma}_{j}\right]$. Substituting in Eq. (10) we then obtain

$$
\begin{aligned}
R_{i j}(t) & =\frac{1}{2} \sum_{l=1}^{n} G^{(l)}(t) \operatorname{Tr}\left[Q_{l-1}^{\dagger}\left\{U_{c}^{\dagger}\left(t, t_{l-1}\right) \hat{\sigma}_{i} U_{c}\left(t, t_{l-1}\right)\right\} Q_{l-1} \hat{\sigma}_{j}\right] \\
& =\frac{1}{2} \sum_{l=1}^{n} G^{(l)}(t) \operatorname{Tr}\left[Q_{l-1}^{\dagger}\left\{\sum_{k=x, y, z} R_{i k}^{P_{l}}\left(t-t_{l-1}\right) \hat{\sigma}_{k}\right\} Q_{l-1} \hat{\sigma}_{j}\right],
\end{aligned}
$$

where we have used Eq. (76) to re-express $U_{c}^{\dagger}\left(t, t_{l-1}\right) \hat{\sigma}_{i} U_{c}\left(t, t_{l-1}\right)$ in terms of the $R_{i j}^{P_{l}}$. Using the linearity of the trace operation and recalling the definition of the of the Control History Matrix elements $\Lambda_{i j}^{l-1}$ from Eq. (80), we then obtain

$$
\begin{aligned}
R_{i j}(t) & =\frac{1}{2} \sum_{k=x, y, z} \sum_{l=1}^{n} G^{(l)}(t) \operatorname{Tr}\left[R_{i k}^{P_{l}}\left(t-t_{l-1}\right) Q_{l-1}^{\dagger} \hat{\sigma}_{k} Q_{l-1} \hat{\sigma}_{j}\right] \\
& =\sum_{k=x, y, z} \sum_{l=1}^{n} G^{(l)}(t) R_{i k}^{P_{l}}\left(t-t_{l-1}\right)\left\{\frac{1}{2} \operatorname{Tr}\left[Q_{l-1}^{\dagger} \hat{\sigma}_{k} Q_{l-1} \hat{\sigma}_{j}\right]\right\} \\
& \equiv \sum_{l=1}^{n} G^{(l)}(t) \sum_{k=x, y, z}\left\{R_{i k}^{P_{l}}\left(t-t_{l-1}\right)\right\}\left\{\Lambda_{k j}^{(l-1)}\right\} .
\end{aligned}
$$

Hence, by definition of matrix multiplication, the time domain total control matrix takes the form

$$
\mathbf{R}(t)=\sum_{l=1}^{n} G^{(l)}(t) \mathbf{R}^{P_{l}}\left(t-t_{l-1}\right) \mathbf{\Lambda}^{(l-1)} .
$$


We move to the frequency domain by performing the integral transform on $\mathbf{R}(t)$ defined by

$$
\begin{aligned}
\mathbf{R}(\omega) & :=-i \omega \int_{0}^{\tau} d t e^{i \omega t} \mathbf{R}(t) \\
& =-i \omega \sum_{l=1}^{n}\left\{\int_{0}^{\tau} d t e^{i \omega t} G^{(l)}(t) \mathbf{R}^{P_{l}}\left(t-t_{l-1}\right)\right\} \boldsymbol{\Lambda}^{(l-1)} \\
& =-i \omega \sum_{l=1}^{n}\left\{\int_{t_{l-1}}^{t_{l}} d t e^{i \omega t} \mathbf{R}^{P_{l}}\left(t-t_{l-1}\right)\right\} \boldsymbol{\Lambda}^{(l-1)} \\
& =\sum_{l=1}^{n} e^{i \omega t_{l-1}}\left\{-i \omega \int_{0}^{\tau_{l}} d t^{\prime} e^{i \omega t^{\prime}} \mathbf{R}^{P_{l}}\left(t^{\prime}\right)\right\} \boldsymbol{\Lambda}^{(l-1)} .
\end{aligned}
$$

In the last line we have performed a change of variables using $t^{\prime}=t-t_{l-1}, \forall l \in\{1, \ldots, n\}$. The frequency-domain Total Control Matrix thus takes the computational form

$$
\mathbf{R}(\omega)=\sum_{l=1}^{n} e^{i \omega t_{l-1}} \mathbf{R}^{P_{l}}(\omega) \mathbf{\Lambda}^{(l-1)},
$$

where we have defined the frequency-domain Local Control Matrix by

$$
\mathbf{R}^{P_{l}}(\omega):=-i \omega \int_{0}^{\tau_{l}} d t^{\prime} e^{i \omega t^{\prime}} \mathbf{R}^{P_{l}}\left(t^{\prime}\right)
$$

The matrix elements of $\mathbf{R}^{P_{l}}(\omega)$ are derived as functions of the control sequence in the section bellow.

\section{Local Control Matrix elements}

As defined in Eq. (79), the matrix elements of the time-domain Local Control Matrix are given by $R_{i j}^{P_{l}}\left(t-t_{l-1}\right)=\frac{1}{2} \operatorname{Tr}\left[U_{c}^{\dagger}\left(t, t_{l-1}\right) \hat{\sigma}_{i} U_{c}\left(t, t_{l-1}\right) \hat{\sigma}_{j}\right]$, where $U_{c}\left(t, t_{l-1}\right) \equiv \exp \left[-i \frac{\Omega_{l}}{2} \hat{\sigma}_{\phi_{l}}(t-\right.$ $\left.t_{l-1}\right)$ ]. The frequency domain representation $\mathbf{R}^{P_{l}}(\omega)$ then follows from the integral transform defined by Eq. (128), with matrix elements expressed as functions of the control parameters $\left\{\Omega_{l}, \tau_{l}, \phi_{l}\right\}$. These matrix elements take the form

$$
\begin{aligned}
& R_{x x}^{P_{l}}(\omega)=\cos ^{2}\left(\phi_{l}\right)\left[1-e^{i \omega \tau_{l}}\right]+\frac{\omega \sin ^{2}\left(\phi_{l}\right)}{\omega^{2}-\Omega_{l}^{2}} V_{l}(\omega), \\
& R_{x y}^{P_{l}}(\omega)=\frac{1}{2} \sin \left(2 \phi_{l}\right)\left[1-e^{i \omega \tau_{l}}\right]-\frac{\omega \sin \left(2 \phi_{l}\right)}{2\left(\omega^{2}-\Omega_{l}^{2}\right)} V_{l}(\omega), \\
& R_{x z}^{P_{l}}(\omega)=-\frac{\omega}{\omega^{2}-\Omega_{l}^{2}} \sin \left(\phi_{l}\right) B_{l}(\omega), \\
& R_{y x}^{P_{l}}(\omega)=\frac{1}{2} \sin \left(2 \phi_{l}\right)\left[1-e^{i \omega \tau_{l}}\right]-\frac{\omega \sin \left(2 \phi_{l}\right)}{2\left(\omega^{2}-\Omega_{l}^{2}\right)} V_{l}(\omega), \\
& R_{y y}^{P_{l}}(\omega)=\sin ^{2}\left(\phi_{l}\right)\left[1-e^{i \omega \tau_{l}}\right]+\frac{\omega \cos ^{2}\left(\phi_{l}\right)}{\omega^{2}-\Omega_{l}^{2}} V_{l}(\omega), \\
& R_{y z}^{P_{l}}(\omega)=\frac{\omega}{\omega^{2}-\Omega_{l}^{2}} \cos \left(\phi_{l}\right) B_{l}(\omega),
\end{aligned}
$$




$$
\begin{aligned}
& R_{z x}^{P_{l}}(\omega)=\frac{\omega}{\omega^{2}-\Omega_{l}^{2}} \sin \left(\phi_{l}\right) B_{l}(\omega) \\
& R_{z y}^{P_{l}}(\omega)=-\frac{\omega}{\omega^{2}-\Omega_{l}^{2}} \cos \left(\phi_{l}\right) B_{l}(\omega) \\
& R_{z z}^{P_{l}}(\omega)=\frac{\omega}{\omega^{2}-\Omega_{l}^{2}} V_{l}(\omega)
\end{aligned}
$$

where we have defined

$$
\begin{aligned}
& V_{l}(\omega):=\left[i \Omega_{l} e^{i \omega \tau_{l}} \sin \left(\Omega_{l} \tau_{l}\right)-\omega e^{i \omega \tau_{l}} \cos \left(\Omega_{l} \tau_{l}\right)+\omega\right] \\
& B_{l}(\omega):=\left[i \Omega_{l} e^{i \omega \tau_{l}} \cos \left(\Omega_{l} \tau_{l}\right)+\omega e^{i \omega \tau_{l}} \sin \left(\Omega_{l} \tau_{l}\right)-i \Omega_{l}\right]
\end{aligned}
$$

\section{Amplitude Control Vector}

The computational form for the frequency-domain Amplitude Control Vector $\mathbf{R}^{(\Omega)}(\omega)$ follows from substituting Eq. (90) into Eq. (111), yielding

$$
\begin{aligned}
\mathbf{R}^{(\Omega)}(\omega) & :=-i \omega \int_{0}^{\tau} d t e^{i \omega t} \mathbf{R}^{(\Omega)}(t) \\
& =-i \omega\left\{\int_{0}^{\tau} d t e^{i \omega t} \sum_{l=1}^{n} G^{(l)}(t)\right\} \overrightarrow{\mathbb{T}}^{(l)} \Lambda^{(l-1)} \\
& =-i \omega \sum_{l=1}^{n}\left\{\int_{t_{l-1}}^{t_{l}} d t e^{i \omega t}\right\} \overrightarrow{\mathbb{T}}^{(l)} \Lambda^{(l-1)} \\
& =-i \omega \sum_{l=1}^{n} \frac{1}{i \omega}\left[e^{i \omega t_{l}}-e^{\left.i \omega t_{l-1}\right] \overrightarrow{\mathbb{T}}^{(l)}} \Lambda^{(l-1)}\right. \\
& =\sum_{l=1}^{n}\left[e^{i \omega t_{l-1}}-e^{i \omega t_{l}}\right] \overrightarrow{\mathbb{T}}^{(l)} \Lambda^{(l-1)} .
\end{aligned}
$$

\section{Appendix C: Walsh Rotary Spin Echo derivations}

The $\mathrm{WRSE}_{k}$ sequence is defined by the phase modulation $\phi(t)=\phi_{0}+\frac{\pi}{2}(1-y(t))$, where $y(t) \in\{ \pm 1\}$. Referring to Eq. (6), however, the spin operator $\hat{\sigma}_{\phi(t)}$ satisfies

$$
\hat{\sigma}_{\phi_{0}+\pi}=-\hat{\sigma}_{\phi_{0}} \quad \Longleftrightarrow \quad \hat{\sigma}_{\phi_{0}+\frac{\pi}{2}(1-y(t))}=y(t) \hat{\sigma}_{\phi_{0}}
$$

Consequently the sign-inversion may be absorbed into a modulated Rabi-rate defined by $\Omega_{y}(t):=y(t) \Omega_{0}$. The sequence is then conveniently recast as amplitude modulation with constant phase $\phi_{0}$. Defining $y(t):=\operatorname{PAL}_{k}(t / \tau)$ we therefore obtain the Walsh synthesis $\Omega_{k}(t)=\Omega_{0} \mathrm{PAL}_{k}(t / \tau)$ consisting of a single Walsh function. Referring to Eq. (31) the associated amplitude modulation is thus given by

$$
\overrightarrow{\boldsymbol{\Omega}}=H_{\mathcal{M}} \tilde{\mathbf{X}}=\Omega_{0} \mathbf{P}_{\mathcal{M}}^{(k)}, \quad \mathcal{M}=2^{m(k)}
$$

where $\tilde{X}_{i(k)}=\Omega_{0}$ is the only nonzero element of $\tilde{\mathbf{X}}$ and, as in Section $5.1, \mathbf{P}_{\mathcal{M}}^{(k)}:=$ $\left(P_{1}^{(k)}, P_{2}^{(k)}, \ldots, P_{\mathcal{M}}^{(k)}\right)^{T}$ defines the $i(k)$ th column of $H_{\mathcal{M}}$. Eq. (46) is then more conveniently 
re-expressed

$$
\mathrm{WRSE}_{k} \equiv \prod_{l=1}^{\mathcal{M}(k)} \exp \left(i \frac{P_{l}^{(k)} \Omega_{0}}{2} \tau_{\mathcal{M}} \hat{\sigma}_{\phi_{0}}\right) .
$$

In the next section we use this form to analyze the amplitude noise filtering properties. The subsequent section treats the filtering properties in the dephasing quadrature.

\section{WRSE $_{k}$ as amplitude noise filters}

Referring to Eq. (147), the rotation operator $\hat{\sigma}_{\phi_{0}}$ for $\mathrm{WRSE}_{k}$ is treated as fixed across all pulses. Thus it commutes with $P_{l}$ and, consequently, with $Q_{l}=P_{l} P_{l-1} \cdots P_{0} \quad \forall l \in$ $\{1, \ldots, \mathcal{M}(k)\}$. It follows $Q_{l-1}^{\dagger} \hat{\sigma}_{\phi_{0}} Q_{l-1}=\hat{\sigma}_{\phi_{0}}$ which, post-multiplying by $\hat{\sigma}_{k}$ and taking the trace of both sides, yields the identity

$$
\frac{1}{2} \operatorname{Tr}\left[\hat{\sigma}_{\phi_{0}} \hat{\sigma}_{k}\right]=\frac{1}{2} \operatorname{Tr}\left[Q_{l-1}^{\dagger} \hat{\sigma}_{\phi_{0}} Q_{l-1} \hat{\sigma}_{k}\right], \quad k \in\{x, y, z\} .
$$

The LHS expands to $\delta_{x k} \cos \left(\phi_{0}\right)+\delta_{y k} \sin \left(\phi_{0}\right)$ (where $\delta_{l k}$ is the Kronecker delta), and the RHS expands to $\cos \left(\phi_{0}\right) \Lambda_{x k}^{(l-1)}+\sin \left(\phi_{0}\right) \Lambda_{y k}^{(l-1)}$ (using the definition of the Pulse History Matrix in Eq. (80)). We thus obtain the following three identities $\forall \phi_{0}$ :

$$
\begin{array}{ll}
k=x: & \cos \left(\phi_{0}\right) \Lambda_{x x}^{(l-1)}+\sin \left(\phi_{0}\right) \Lambda_{y x}^{(l-1)}=\cos \left(\phi_{0}\right), \\
k=y: & \cos \left(\phi_{0}\right) \Lambda_{x y}^{(l-1)}+\sin \left(\phi_{0}\right) \Lambda_{y y}^{(l-1)}=\sin \left(\phi_{0}\right), \\
k=z: & \cos \left(\phi_{0}\right) \Lambda_{x z}^{(l-1)}+\sin \left(\phi_{0}\right) \Lambda_{y z}^{(l-1)}=0 .
\end{array}
$$

Now, setting $\Omega_{l} \equiv P_{l}^{(k)} \Omega_{0}$ the Projection Vector defined in Eq. (18) becomes $\overrightarrow{\mathbb{T}}^{(l)}=\left(\Omega_{0} P_{l}^{(k)} /\right.$ $2)\left[\cos \left(\phi_{0}\right), \sin \left(\phi_{0}\right), 0\right]$, in which case

$$
\begin{aligned}
& \overrightarrow{\mathbb{T}}^{(l)} \boldsymbol{\Lambda}^{(l-1)}=\frac{\Omega_{0}}{2} P_{l}^{(k)}\left\{\cos \left(\phi_{0}\right) \boldsymbol{\Lambda}_{x}^{(l-1)}+\sin \left(\phi_{0}\right) \boldsymbol{\Lambda}_{y}^{(l-1)}\right\} \\
& =\frac{\Omega_{0}}{2} P_{l}^{(k)}\left[\begin{array}{l}
\cos \left(\phi_{0}\right) \Lambda_{x x}^{(l-1)}+\sin \left(\phi_{0}\right) \Lambda_{y x}^{(l-1)} \\
\cos \left(\phi_{0}\right) \Lambda_{x y}^{(l-1)}+\sin \left(\phi_{0}\right) \Lambda_{y y}^{(l-1)} \\
\cos \left(\phi_{0}\right) \Lambda_{x z}^{(l-1)}+\sin \left(\phi_{0}\right) \Lambda_{y z}^{(l-1)}
\end{array}\right]^{T} \\
& =\frac{\Omega_{0}}{2} P_{l}^{(k)}\left[\cos \left(\phi_{0}\right), \quad \sin \left(\phi_{0}\right), \quad 0\right],
\end{aligned}
$$

where in the last equality we have used the identities derived above in Eqs. (149), (150) and (151). Using Eq. (16) we therefore obtain

$$
\mathbf{R}^{(\Omega)}(\omega)=\frac{\Omega_{0}}{2} \sum_{l=1}^{\mathcal{M}} P_{l}^{(k)}\left[e^{i \omega t_{l-1}}-e^{i \omega t_{l}}\right]\left[\begin{array}{c}
\cos \left(\phi_{0}\right) \\
\sin \left(\phi_{0}\right) \\
0
\end{array}\right]^{T},
$$

where $t_{l}=l \tau / \mathcal{M}(k)$. From Eq. (14) the amplitude filter-transfer function therefore becomes

$$
F_{\Omega}(\omega)=\frac{\Omega^{2}}{4}\left|\sum_{l=1}^{\mathcal{M}} P_{l}^{(k)}\left[e^{i \omega t_{l-1}}-e^{i \omega t_{l}}\right]\right|^{2},
$$


where, on taking the modulus square, the $\phi_{0}$ dependence amounts to $\cos \left(\phi_{0}\right)^{2}+\sin \left(\phi_{0}\right)^{2}=$ 1 and hence vanishes.

\section{$\mathrm{WRSE}_{k}$ as dephasing noise filters}

For a general WRSE ${ }_{k}$ sequence, one can show the first-order Taylor coefficient for $F_{z}(\omega)$ takes the analytic form

$$
C_{2}^{(z)}\left(\Omega_{0} ; k\right)=\operatorname{sinc}^{2}\left(\frac{\Omega_{0}}{2^{\kappa(k)}}\right), \quad \kappa(k)= \begin{cases}m(k) & \text { if } r(k) \neq 1, \\ m(k)+1 & \text { if } r(k)=1\end{cases}
$$

yielding the family of zeros $Z_{2}^{(k)}=\left\{2^{\kappa(k)} \pi q \mid q \in \mathbb{N}\right\}$. Hence it is always possible to produce a first-order filter with $(p-1)=1$ by setting $\Omega_{0} \in Z_{2}^{(k)}$. Higher-order filters for dephasing noise - that is, such that $(p-1)>1$ - then correspond to some $\eta$ satisfying Eq. (49) such that $\eta \in Z_{2}^{(k)}$. Although a general analytical form for these higher-order coefficients is not easy to express ${ }^{\mathrm{n}}$ we may still make progress, however, by simply substituting in the candidate values $\Omega_{0}=2^{\kappa(k)} \pi q$ and determining which $q \in \mathbb{N}$ produce concurrent zeros of the $C_{2 j}^{(z)}\left(\Omega_{0}\right)$. As a representative example we study the particular case for $\mathrm{WRSE}_{3}$, deriving the coefficients

$$
\begin{aligned}
C_{4}^{(z)}\left(\Omega_{0} ; 3\right)= & \frac{1}{\Omega_{0}^{4}}\left[\left(\Omega_{0}^{2}-16\right) \cos \frac{\Omega_{0}}{2}-2 \Omega_{0}^{2} \cos \frac{\Omega_{0}}{4}-8 \Omega_{0} \sin \frac{\Omega_{0}}{4}+\left(\Omega_{0}^{2}+16\right)\right], \\
C_{6}^{(z)}\left(\Omega_{0} ; 3\right)= & \frac{1}{48 \Omega_{0}^{6}}\left[\left(\Omega_{0}^{4}-96 \Omega_{0}^{2}+1,152\right) \cos \frac{\Omega_{0}}{2}-\left(5 \Omega_{0}^{4}-192 \Omega_{0}^{2}\right) \cos \frac{\Omega_{0}}{4}\right. \\
& \left.-\left(28 \Omega_{0}^{3}-768 \Omega_{0}\right) \sin \frac{\Omega_{0}}{4}+4\left(\Omega_{0}^{4}-36 \Omega_{0}^{2}-288\right)\right] .
\end{aligned}
$$

From above, the choice $k=3$ implies $\kappa(k)=2$ and consequently the candidate zeros take the form $\Omega_{0}=4 \pi q$. Substituting into the above expressions yields

$$
\begin{aligned}
& C_{4}^{(z)}(4 \pi q ; 3)=\frac{1-(-1)^{q}}{8 q^{2} \pi^{2}}, \quad q \in \mathbb{N}, \\
& C_{6}^{(z)}(4 \pi q ; 3)= \begin{cases}\frac{1}{(8 \pi q)^{4}} & \text { if } q \text { even, } \\
\frac{27-10 \pi^{2}(1+2 q)^{2}}{7 \pi^{4}(1+2 q)^{4}} & \text { if } q \text { odd. }\end{cases}
\end{aligned}
$$

Thus $q$ must be even to ensure $C_{4}^{(z)}=0$. However this choice implies $C_{6}^{(z)}>0$ (in fact $C_{6}^{(z)}>0$ for any choice of $q$ ) and it follows $\mathrm{WRSE}_{3}$ is at maximum a second-order filter. In Figure 6(a) we plot $C_{2 j}^{(z)}\left(\Omega_{0} ; 3\right), j \in\{1,2,3\}$ showing the existence of concurrent zeros only for $j \in\{1,2\}$. The inset shows, as a representative case, the behaviour near $\Omega_{0}=8 \pi$. In Figure 6(b) we plot $F_{z}(\omega)$ setting $\Omega_{0}=2 \pi q, q \in\{1, \ldots, 8\}$, showing values for which first and second-order filtering is achieved. Repeating this general procedure for other values of $k$ we find similar results and conclude the $\mathrm{WRSE}_{k}$ family are capable of up to secondorder filtering against dephasing noise. 
Authors' contributions

All authors contributed equally to the writing of this paper. All authors read and approved the final manuscript.

\section{Acknowledgements}

We thank K Brown, JT Merrill and L Viola for useful discussions. This work partially supported by the US Army Research Office under Contract Number W911NF-11-1-0068, and the Australian Research Council Centre of Excellence for Engineered Quantum Systems CE110001013.

\section{Endnotes}

a The assumption of independence is reasonable, for instance, in the case of a driving field where random fluctuations in frequency and amplitude arise from different physical processes. A general model including correlations between noise processes is possible, however, following the approach outlined by Green et al. [49].

b The first-order approximation has recently been experimentally tested and demonstrated to produce good agreement in the weak noise limit [37]. For the noise field $\beta(t)$, this regime is sufficiently characterized by requiring $\xi^{2} \ll 1$, where the smallness parameter is defined by $\xi^{2} \equiv\left\langle\beta^{2}(t)\right\rangle \tau^{2} \equiv \tau^{2} \int_{-\infty}^{+\infty} d \omega S_{\beta}(\omega)$ [48]. The condition $\xi^{2}<1$ is also required for the Magnus series to formally converge.

c For a resonantly driven qubit $\phi_{l}$ is the phase of the driving field and $\Omega_{\text {/ }}$ is linearly proportional to the driving amplitude.

d This effect is captured by the multiple slopes in Figure 4(h) which clearly show the difference between the asymptotic zero-frequency roll-off and the local slope over targeted regions $\left[\omega_{L}, \omega_{c}\right]$ in the stopband.

e All stopbands 'turn on' with a finite response, the functional form of which determines the filter order and the effectiveness of noise suppression. In the stopband this is quantified by the slope, or roll off in the language of filter design.

$f$ This can be shown by Taylor expanding the amplitude noise filter function $F_{\Omega}\left(\omega \tau ; \boldsymbol{\Gamma}_{n}^{\mathrm{AMF}}\right)$ and deriving the result that $C_{2}^{(\Omega)}\left(\boldsymbol{\Gamma}_{n}^{\mathrm{AMF}}\right)=\frac{1}{4}\left(\sum_{l=1}^{n} \theta_{l}\right)^{2}$.

g We use the vectors $\mathbf{X} \equiv\left(X_{0}, X_{1}, \ldots, X_{N}\right)$ and $\mathbf{Y} \equiv\left(Y_{0}, Y_{1}, \ldots, Y_{N}\right)$ to compactly write the Paley ordered Walsh spectra implied by Eq. (29) in synthesizing $\Omega(t)$ and $\phi(t)$.

h Pulse periods taking non-integer multiples values of $\tau_{\min }$ then have intrinsic conflict with implementation in discretized time via digital control, giving rise to residual errors.

i Our studies have not produced proof that this symmetry is strictly necessary. In fact for WPMFs it is not required. However WAMF constructions possessing time-reversal symmetry do appear to yield results more readily, and all WAMFs we have discovered have this property.

j Maximizing the number of Rademacher functions does not correspond to maximizing the switching rate of $\operatorname{PAL}_{k}(x)$. In fact, for a given $m(k)$ the maximum switching rate for $\operatorname{PAL}_{k}(x)$ corresponds to $k=2^{m(k)-1}$, which consists of the single Rademacher function $R_{m(k)-1}(x)$.

$k$ These points may also be derived using Nelder-Mead optimization of $A_{z}\left(X_{0} ; X_{3}\right)$ over the two-dimensional domain. This method is useful for more complex constructions (see Section 6.2) where spectral optimization becomes a more multi-dimensional task.

I Since the $\mathrm{WAMF}_{0.7}^{(2)}$ gates in Figure 4 were derived by optimizing the cost function over local regions in the stopband, the asymptotic filter order $(p-1)$ associated with Taylor expanding $F_{z}(\omega)$ about $\omega=0$ is not a meaningful descriptor of these filters. Hence we do not expect $C_{2,4}^{(z)}=0$ and do not pursue such a calculation. Instead the instantaneous filter order is used.

$m$ This is valid since $\Phi(\tau)$ belongs to the Lie algebra of $S U(2)$, inheriting this property from the toggling frame Hamiltonians from which it is derived.

$\mathrm{n}$ The higher-order $C_{2 j}^{(k)}$ involve terms oscillating at multiple frequencies and have nontrivial dependencies on $\Omega_{0}$ Their zeros must in general be determined numerically.

Received: 13 December 2014 Accepted: 19 March 2015 Published online: 14 May 2015

\section{References}

1. Van Harlingen DJ, Plourde BLT, Robertson TL, Reichardt PA, Clarke J. Decoherence in flux qubits due to $1 / f$ noise in Josephson junctions. In: Leggett A, Ruggiero B, Silvestini P, editors. Quantum computing and quantum bits in mesoscopic systems. New York: Kluwer Academic; 2004. p. 171-84.

2. Faoro L, Viola L. Dynamical suppression of 1/f noise processes in qubit systems. Phys Rev Lett. 2004;92:117905.

3. Bylander J, Gustavsson S, Yan F, Yoshihara F, Harrabi K, Fitch G, Cory DG, Nakamura Y, Tsai J-S, Oliver WD. Noise spectroscopy through dynamical decoupling with a superconducting flux qubit. Nat Phys. 2011;7:565-70.

4. Zhang W, Dobrovitski W, Santos LF, Viola L, Harmon BN. Dynamical control of electron spin coherence in a quantum dot: a theoretical study. Phys Rev B. 2007;75:201302.

5. Biercuk MJ, Uys H, VanDevender AP, Shiga N, Itano WM, Bollinger JJ. High-fidelity quantum control using ion crystals in a Penning trap. Quantum Inf Comput. 2009;9:920-49.

6. Rutman J. Characterization of phase and frequency instabilities in precision frequency sources: fifteen years of progress. Proc IEEE. 1978;66:1048.

7. Tarn TJ, Huang G, Clark JW. Modelling of quantum mechanical control systems. Math Model. 1980;1:109.

8. Clark JW, Lucarelli DG, Tarn TJ. Control of quantum systems. Int J Mod Phys B. 2003;17:5397.

9. Bouten L, Van Handel R, James MR. An introduction to quantum filtering. SIAM J Control Optim. 2007;46:2199.

10. Nurdin HI, James MR, Petersen IR. Coherent quantum LGQ control. Automatica. 2009;45:1837.

11. Viola L, Lloyd S, Knill E. Universal control of decoupled quantum systems. Phys Rev Lett. 1999;83:4888.

12. Ticozzi F, Nishio K, Altafini C. Stabilization of stochastic quantum dynamics via open and closed loop control. IEEE Trans Autom Control. 2013;58:74-85. 
13. Lidar DA, Brun TA. Quantum error correction. New York: Cambridge University Press; 2013.

14. Jones NC, Van Meter R, Fowler AG, McMahon PL, Kim J, Ladd TD, Yamamoto Y. Layered architecture for quantum computing. Phys Rev X. 2012;2:031007.

15. Biercuk MJ, Uys H, VanDevender AP, Shiga N, Itano WM, Bollinger JJ. Optimized dynamical decoupling in a mode quantum memory. Nature. 2009;458:996.

16. Khodjasteh K, Sastrawan J, Hayes D, Green TJ, Biercuk MJ, Viola L. Designing a practical high-fidelity long-time quantum memory. Nat Commun. 2013;4:2045.

17. Du J, Rong X, Zhao N, Wang Y, Yang J, Liu RB. Preserving electron spin coherence in solids by optimal dynamical decoupling. Nature. 2009;461:1265.

18. Sagi Y, Almog I, Davidson N. Process tomography of dynamical decoupling in a dense cold atomic ensemble. Phys Rev Lett. 2010;105:053201.

19. Khodjasteh K, Viola L. Dynamically error-corrected gates for universal quantum computation. Phys Rev Lett. 2009;102:080501.

20. Khodjasteh K, Lidar DA, Viola L. Arbitrarily accurate dynamical control in open quantum systems. Phys Rev Lett. 2010;104:090501.

21. Wang X, Bishop LS, Kestner JP, Barnes E, Sun K, Das Sarma S. Composite pulses for robust universal control of singlet-triplet qubits. Nat Commun. 2012;3:997.

22. Souza AM, Álvarez GA, Suter D. Experimental protection of quantum gates against decoherence and control errors. Phys Rev A. 2012;86:050301.

23. van der Sar T, Wang ZH, Blok MS, Bernien H, Taminiau TH, Toyli DM, Lidar DA, Awschalom DD, Hanson R, Dobrovitski W. Decoherence-protected quantum gates for a hybrid solid-state spin register. Nature. 2012;484:82-6.

24. Liu G-Q, Po HC, Du J, Liu RB, Pan X-Y. Noise-resilient quantum evolution steered by dynamical decoupling. Nat Commun. 2013;4:2254

25. Fauseweh B, Pasini S, Uhrig GS. Frequency-modulated pulses for quantum bits coupled to time-dependent baths. Phys Rev A. 2012;85:022310.

26. Vandersypen LMK, Chuang IL. NMR techniques for quantum control and computation. Rev Mod Phys. 2005;76:1037.

27. Merrill JT, Brown KR. Progress in compensating pulse sequences for quantum computation. arXiv:1203.6392 (2012).

28. Kabytayev C, Green TJ, Khodjasteh K, Biercuk MJ, Viola L, Brown KR. Robustness of composite pulses to time-dependent control noise. Phys Rev A. 2014;90:012316.

29. Viola L, Knill E. Robust dynamical decoupling of quantum systems with bounded controls. Phys Rev Lett. 2003:90:037901.

30. Jones NC, Ladd TD, Fong BH. Dynamical decoupling of a qubit with always-on control fields. New J Phys. 2012;14:093045

31. Cai J-M, Naydenov B, Pfeiffer R, McGuinness LP, Jahnke KD, Jelezko F, Plenio MB, Retzker A. Robust dynamical decoupling with concatenated continuous driving. New J Phys. 2012;14:113023.

32. Fanchini FF, Hornos JEM, Napolitano RdJ. Continuously decoupling single-qubit operations from a perturbing thermal bath of scalar bosons. Phys Rev A. 2007;75:022329.

33. Xu X, Wang Z, Duan C, Huang P, Wang P, Wang Y, Xu N, Kong X, Shi F, Rong X, Du J. Coherence-protected quantum gate by continuous dynamical decoupling in diamond. Phys Rev Lett. 2012;109:070502.

34. Bermudez A, Schmidt PO, Plenio MB, Retzker A. Robust trapped-ion quantum logic gates by continuous dynamical decoupling. Phys Rev A. 2012;85:040302.

35. Chaudhry AZ, Gong J. Decoherence control: universal protection of two-qubit states and two-qubit gates using continuous driving fields. Phys Rev A. 2012;85:012315.

36. Lemmer A, Bermudez A, Plenio MB. Driven geometric phase gates with trapped ions. New J Phys. 2013;15:083001

37. Soare A, Ball H, Hayes D, Sastrawan J, Jarratt MC, McLoughlin JJ, Zhen X, Green TJ, Biercuk MJ. Experimental noise filtering by quantum control. Nat Phys. 2014;10:825-9.

38. Silva GP, Viola L. A general transfer-function approach to noise filtering in open-loop quantum control. arXiv:1408.3836 (2014)

39. Kofman AG, Kurizki G. Universal dynamical control of quantum mechanical decay: modulation of the coupling to the continuum. Phys Rev Lett. 2001;87:270405.

40. Kofman AG, Kurizki G. Unified theory of dynamically suppressed qubit decoherence in thermal baths. Phys Rev Lett. 2004;93:130406

41. Girod B, Rabenstein R, Stenger A. Signals and systems. New York: Wiley; 2001

42. Biercuk MJ, Doherty AC, Uys H. Dynamical decoupling sequence construction as a filter-design problem. J Phys B. 2011:44:154002

43. Martinis JM, Nam S, Aumentado J, Lang KM, Urbina C. Decoherence of a superconducting qubit due to bias noise. Phys Rev B. 2003;67:094510

44. Kuopanportti P, Mottonen M, Bergholm V, Saira O-P, Zhang J, Whaley KB. Suppression of $1 / f^{\alpha}$ noise in one-qubit systems. Phys Rev A. 2008;77:032334

45. Uys H, Biercuk MJ, Bollinger JJ. Optimized noise filtration through dynamical decoupling. Phys Rev Lett. 2009;103:040501.

46. Uhrig GS. Keeping a quantum bit alive by optimized pi-pulse sequences. Phys Rev Lett. 2007:98:100504.

47. Cywinski L, Lutchyn RM, Nave CP, Sarma SD. How to enhance dephasing time in superconducting qubits. Phys Rev B. 2008;77:174509.

48. Green TJ, Uys H, Biercuk MJ. High-order noise filtering in nontrivial quantum logic gates. Phys Rev Lett. 2012;109:020501.

49. Green TJ, Sastrawan J, Uys H, Biercuk MJ. Arbitrary quantum control of qubits in the presence of universal noise. New J Phys. 2013;15:095004

50. Hayes D, Clark SM, Debnath S, Hucul D, Inlek IV, Lee KW, Quraishi Q, Monroe C. Coherent error suppression in multiqubit entangling gates. Phys Rev Lett. 2012;109:020503.

51. Hayes D, Khodjasteh K, Viola L, Biercuk MJ. Reducing sequencing complexity in dynamical quantum error suppression by Walsh modulation. Phys Rev A. 2011;84:062323. 
52. Green TJ, Biercuk MJ. Phase-modulated decoupling and error suppression in qubit-oscillator systems. arXiv:1408.2749 (2014).

53. Owrutsky P, Khaneja N. Control of inhomogeneous ensembles on the Bloch sphere. Phys Rev A. 2012;86:022315.

54. Hodgson TE, Viola L, D'Amico I. Towards optimized suppression of dephasing in systems subject to pulse timing constraints. Phys Rev A. 2010;81:062321.

55. Schumacher B. Sending entanglement through noisy quantum channels. Phys Rev A. 1996:54:2614.

56. Blanes S, Cases F, Oteo JA, Ros J. The Magnus expansion and some of its applications. Phys Rep. 2009;470:151.

57. Magnus W. On the exponential solution of differential equations for a linear operator. Commun Pure Appl Math. $1954 ; 7: 649$.

58. Haeberlen U, Waugh JS. Coherent averaging effects in magnetic resonance. Phys Rev. 1968;175:453.

59. Ernst RR, Bodenhausen GB, Wokaun A. Principles of nuclear magnetic resonance in one and two dimensions. New York: Oxford University Press; 1987.

60. Ajoy A, Álvarez GA, Suter D. Optimal pulse spacing for dynamical decoupling in the presence of a purely-dephasing spin-bath. Phys Rev A. 2011;83:032303.

61. Beauchamp KG. Walsh functions and their applications. London: Academic Press; 1975.

62. Walsh JL. A closed set of normal orthogonal functions. Am J Math. 1923;45:5.

63. Tzafestas SG. Walsh functions in signal and systems analysis and design. New York: Van Nostrand Reinhold; 1985.

64. Cooper A, Magesan E, Yum HN, Cappellaro P. Time-resolved magnetic sensing with electronic spins in diamond. Nat Commun. 2014;5:3141.

65. Harmuth HF. Transmission of information by orthogonal functions. New York: Springer; 1969.

66. Harmuth HF. Applications of Walsh functions in communications. IEEE Spectr. 1969;6:82.

67. Paley R. A remarkable series of orthogonal functions (I). Proc Lond Math Soc. 1932;2:241.

68. Rademacher H. Einige Sätze über Reihen von allgemeinen Orthogonalfunktionen. Math Ann. 1922;87:241.

69. Horadam KJ. Hadamard matrices and their applications. Princeton: Princeton University Press; 2007.

70. Khodjasteh K, Viola L. Dynamical quantum error correction of unitary operations with bounded controls. Phys Rev A. 2009;80:032314.

71. Souza AM, Álvarez GA, Suter D. Effects of time-reversal symmetry in dynamical decoupling. Phys Rev A. 2012;85:032306.

72. Brown KR, Harrow AW, Chuang IL. Arbitrarily accurate composite pulse sequences. Phys Rev A. 2004;70:052318

73. Brown KR, Harrow AW, Chuang IL. Erratum: Arbitrarily accurate composite pulse sequences [Phys. Rev. A 70, 052318 (2004)]. Phys Rev A. 2005;72:039905.

74. Wimperis S. Broadband, narrowband, and passband composite pulses for use in advanced $\{$ NMR $\}$ experiments. J Magn Reson, Ser A. 1994;109:221

75. Yan F, Gustavsson S, Bylander J, Jin X, Yoshihara F, Cory DG, Nakamura Y, Orlando TP, Oliver WD. Rotating-frame relaxation as a noise spectrum analyser of a superconducting qubit undergoing driven evolution. Nat Commun. 2013:4:2337.

76. Gustavsson S, Bylander J, Yan F, Forn-Díaz P, Bolkhovsky V, Braje D, Fitch G, Harrabi K, Lennon D, Miloshi J, Murphy P, Slattery R, Spector S, Turek B, Weir T, Welander PB, Yoshihara F, Cory DG, Nakamura Y, Orlando TP, Oliver WD. Driven dynamics and rotary echo of a qubit tunably coupled to a harmonic oscillator. Phys Rev Lett. 2012;108:170503.

77. Miller SL, Childers D. Probability and random processes with applications to signal processing and communications. Boston: Academic Press; 2012.

\section{Submit your manuscript to a SpringerOpen ${ }^{\circ}$ journal and benefit from:}

- Convenient online submission

Rigorous peer review

- Immediate publication on acceptance

- Open access: articles freely available online

- High visibility within the field

- Retaining the copyright to your article 